\title{
1 Targeted photostimulation uncovers circuit motifs supporting short-term memory
}

2 Kayvon Daie ${ }^{1}$, Karel Svoboda ${ }^{1}$ \& Shaul Druckmann ${ }^{1,2}$

$3{ }^{1}$ Janelia Research Campus, HHMI, Ashburn VA 20147

$4 \quad{ }^{2}$ Stanford University, Stanford CA 94305

5 Correspondence: svobodak@janelia.hhmi.org or shauld@stanford.edu

\section{Abstract}

Short-term memory is associated with persistent neural activity without sustained input, arising from the interactions between neurons with short time constants ${ }^{1,2}$. A variety of neural circuit motifs could account for measured neural activity ${ }^{3-7}$. A mechanistic understanding of the neural circuits supporting short-term memory requires probing network connectivity between functionally characterized neurons ${ }^{8}$. We performed targeted photostimulation of small $(<10)$ groups of neurons, while imaging the response of hundreds of other neurons $s^{9,10}$, in anterior-lateral motor cortex (ALM) of mice performing a delayed response task ${ }^{11}$. Mice were instructed with brief auditory stimuli to make directional movements (lick left or lick right), but only after a three second delay epoch. ALM contains neurons with delay epoch activity that is selective for left or right choices. Targeted photostimulation of groups of neurons during the delay epoch allowed us to observe the functional organization of population activity and recurrent interactions underlying short-term memory. These experiments revealed strong coupling between neurons sharing similar selectivity. Brief photostimulation of functionally related neurons produced changes in activity in sparse subpopulations of nearby neurons that persisted for several seconds following stimulus offset, far outlasting the duration of the perturbation. Photostimulation produced behavioral biases that were predictable based on the selectivity of the perturbed neuronal population. These results suggest that ALM contains multiple intercalated modules, consisting of recurrently coupled neurons, that can independently maintain persistent activity. 
Mice expressing the calcium indicator GCaMP6s ${ }^{12}$ and the light-activated cation channel soma-targeted (ST) ChrimsonR ${ }^{13,14}$ (Extended Data Fig. 1) were trained to discriminate two tones. After a delay epoch lasting three seconds, mice reported the identity of the tone with directional licking (Fig. 1a) ${ }^{15}$. We imaged activity in layer 2/3 (125-250 um deep; typical field of view (FOV), $600 \times 600 \mu \mathrm{m}^{2}$ ) of the left hemisphere of ALM (8 mice, 84 sessions, 324 trials per session; range, 208 - 441 trials; Extended Data Fig. 2). On average, 49 neurons (range, $14-98,75 \% \mathrm{Cl} ; 25 \%$ of the imaged population) per FOV were selective during the delay and early response epochs (mean, 26 right- and 23 left-selective, Extended Data Fig. 3, one-tailed t-test, $\mathrm{p}<0.05)^{16,17}$.

To probe the circuit basis of persistent activity we used two-photon photostimulation of small groups of neurons (Fig. 1b,c) and measured responses in other neurons (Fig. 1d-i). We targeted neurons in groups of eight ('photostimulation group'), which ensured that we could 1) photostimulate sufficient numbers of spatially distributed neurons to potentially alter network activity and 2) observe changes in activity in nontargeted selective neurons, as an indicator of network connectivity. Targeted neurons were photostimulated by scanning the beam over their cell bodies for 3 milliseconds (Extended Data Fig. 4), causing short-latency (mean, $5 \pm 2 \mathrm{~ms}$; mean \pm s.e.m.) spikes (range, $0.2-1.5$ spikes per stimulus) (Extended Data Fig. 5). Neurons in photostimulation groups were photostimulated sequentially, ten times at $31.25 \mathrm{~Hz}$ (total duration, $319 \mathrm{~ms}$; Extended Data Fig. 4). A large proportion of targeted neurons displayed increases in GCaMP6s fluorescence ( $\Delta \mathrm{F} / \mathrm{F}$; mean, 0.43; range, $0.07-0.80,75 \% \mathrm{Cl})$. Photostimuli were applied during the delay epoch (on $33.3 \%$ or $40 \%$ of trials). Multiple (2-5) photostimulation groups 
bioRxiv preprint doi: https://doi.org/10.1101/623785; this version posted April 30, 2019. The copyright holder for this preprint (which was not certified by peer review) is the author/funder. All rights reserved. No reuse allowed without permission.

b

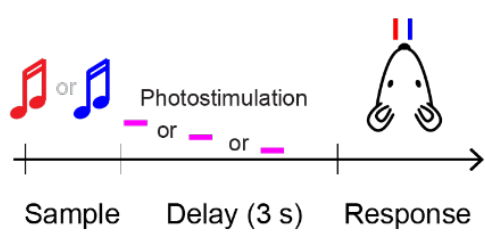

d Photostimulation group 1

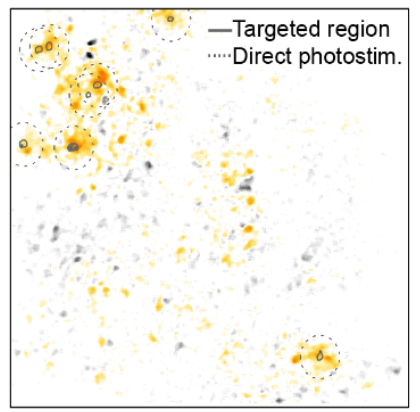

Photostimulation group 2

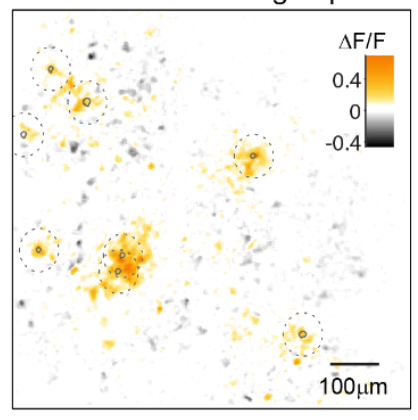

g

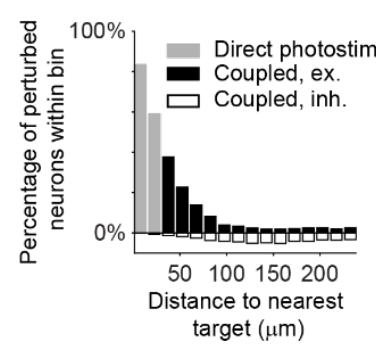

e

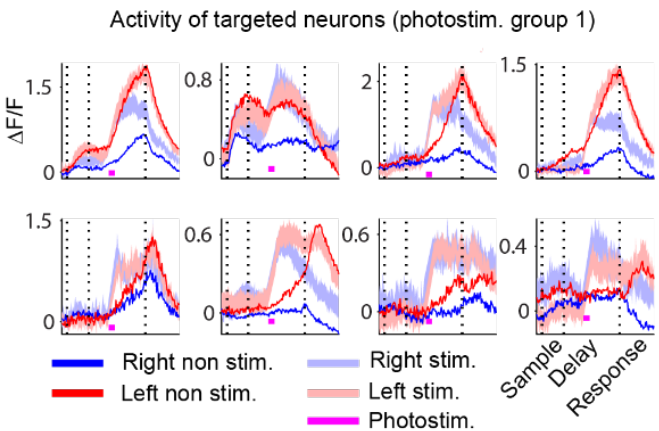

Activity of targeted neurons (photostim. group 2)
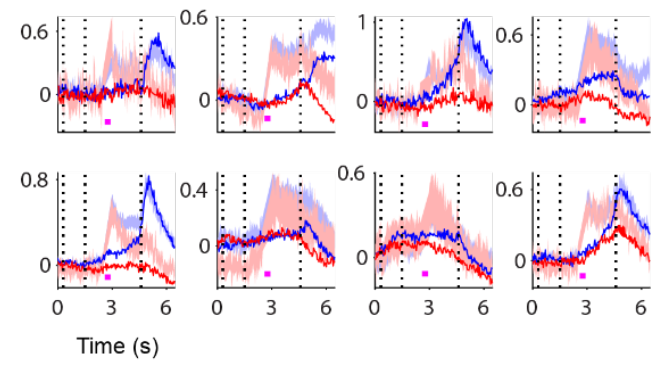

h

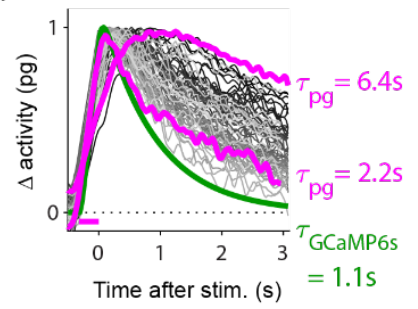

Spatial resolution of photostimulation

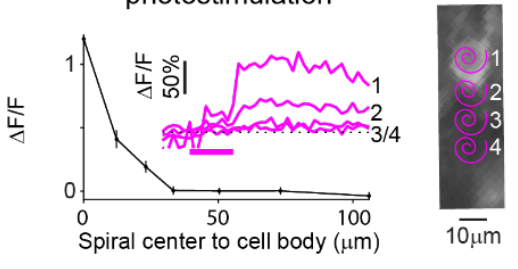

f

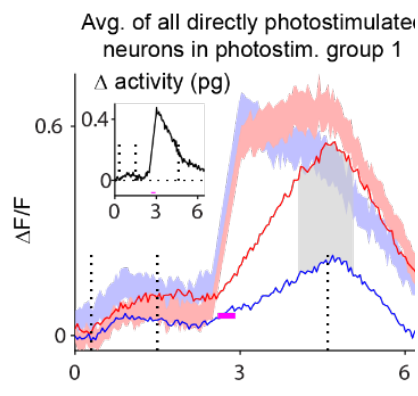

Avg. of all directly photostimulate neurons in photostim. group 2

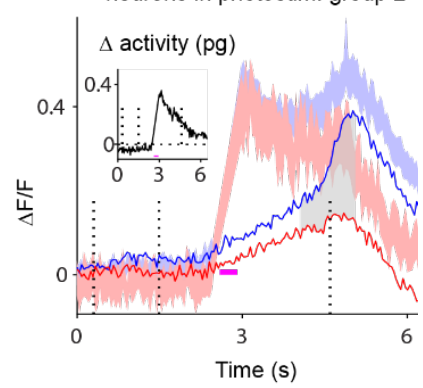

j

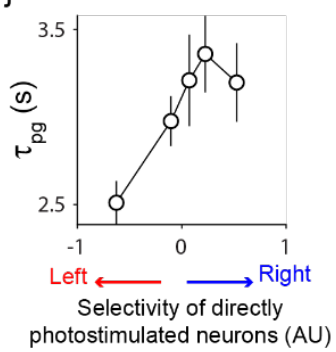

Figure 1: Targeted photostimulation during performance of the delayed-response task. a. Task structure. During the sample epoch, mice were instructed by an auditory cue to lick left or lick right for a water reward. Responses were allowed after a 3 seconds long delay epoch. Photostimuli (magenta bars) were delivered on a random subset of trials during the delay epoch. b. Targeted photostimulation. Two-photon imaging was used to map selectivity of individual neurons during behavior (lick left, red; lick right, blue). Two-photon photostimulation (magenta dashed lines) was used to activate groups of neurons based on their selectivity. c. Measurement of lateral spatial resolution of photostimulation. Left, change in GCaMP6s fluorescence vs. distance between photostimulus (spiral) and imaged neuron (9 neurons). Inset, responses to photostimuli delivered at different distances ( $\mu \mathrm{m} ; 0,12.5,25$ and 37.5) (magenta bar, photostimulation, $320 \mathrm{~ms}$ ). Right, example experiment. (Continued on next page) 
(Continued from previous page) Figure 1: Targeted photostimulation during performance of the delayed-response task. $d$ f. Top, photostimulation group 1; bottom, photostimulation group 2. d, Example photostimulation experiment. Black ROIs indicate targeted neurons. Dashed circles (radius $20 \mu \mathrm{m}$ ) indicate region with neurons that could be receiving significant direct photostimulation. Pixel color indicates the fractional difference in fluorescence between photostimulation and nonphotostimulation trials, averaged over $500 \mathrm{~ms}$ after the photostimulus. (Excited coupled neurons, 22 in group 1, 23 in group 2; Inhibited coupled neurons, 6 in group 1, 7 in group 2; $p<0.05$, one-tailed T-test) e. Fluorescence changes (averaged across correct trials) for each of the eight targeted neurons per photostimulation group, on photostimulation (light traces; error shade = s.e.m.) and non-photostimulation (dark traces) trials (trial types: red, left; blue, right). f. Responses averaged across all directly photostimulated neurons per photostimulation group. Gray shading, average selectivity of directly photostimulated neurons ( $x$-axis in panel $j$ ). Inset, difference in activity between photostimulation and non-photostimulation trials, averaged across all directly photostimulated neurons in a photostimulation group ( $\Delta$ activity pg).g. Percentage of neurons with activity that is directly excited (gray) or coupled (black, excited; white, inhibited) by photostimulation, as a function of distance (from the nearest photostimulation target). h. Decay times $\left(\tau_{\mathrm{pg}}\right)$ of $\Delta$ activity pg (panel $\mathrm{f}$, inset). Traces are color-coded based on decay time (gray lines). Green line, fluorescence decay after a brief burst of activity (Extended Data Fig. 7d). Magenta bar, time of photostimulation. i. Decay time constants of coupled neurons ( $\tau_{\text {coupled }}$ ) vs. $\tau_{p g}$. j. $\tau_{p g} v s$. average selectivity of the directly photostimulated neurons (panel f).

In addition to targeted neurons, cells up to $20 \mu \mathrm{m}$ laterally from the center of the photostimulus could

have been directly photostimulated (Fig. 1c, Extended Data Fig. 6). We refer to activated neurons in this

neighborhood together as 'directly photostimulated' (Fig. 1f). Neural activity more than $30 \mu \mathrm{m}$ from the

targeted neurons changed as well (Fig. 1d). Control experiments show that changes in activity at distances

greater than $30 \mu \mathrm{m}$ from a photostimulus result exclusively from synaptic interactions with the directly

photostimulated population (Fig. 1c, Extended Data Fig. 6). We refer to these neurons as 'coupled'.

Photostimulation groups produced detectable excitation in 20 coupled neurons (range, $4-39$ neurons,

$75 \% \mathrm{Cl}$, two-tailed t-test, $\mathrm{p}<0.05$ ), and inhibition in 6 coupled neurons (range, $0-13$ neurons, $75 \% \mathrm{Cl}$ ) per

FOV. The number of excited or inhibited coupled neurons decreased with distance from the photostimuli

(Fig. 1g) (length constant, $40 \mu \mathrm{m}$ ). Additional coupled neurons were presumably outside of the FOV.

Photostimuli caused transient increases in activity with diverse amplitudes and dynamics (Fig. 1e). On average, photostimulation increased the activity of targeted (Fig. 1e) and directly photostimulated (Fig. 1f) neurons for several seconds (mean, $\tau_{p g}=3.1 \mathrm{~s}$; range, $2.0 \mathrm{~s}-4.6 \mathrm{~s}, 75 \% \mathrm{Cl}$ ). The $\tau_{\mathrm{pg}}$ are much longer

than the decay of GCaMP6s fluorescence expected after a short burst of activity ${ }^{12}\left(\tau_{\text {GCaMPGs }}=1.1 \mathrm{~s}\right.$, Fig. $1 \mathrm{~h}$, green line; Extended Data Fig. 7). These long-lasting changes in fluorescence likely reflect persistent 
bioRxiv preprint doi: https://doi.org/10.1101/623785; this version posted April 30, 2019. The copyright holder for this preprint (which was not certified by peer review) is the author/funder. All rights reserved. No reuse allowed without permission. $\tau_{\mathrm{pg}}$ (Fig. 1i, Pearson correlation $\left.=0.49, p<10^{-5}\right)$, suggesting that photostimulation triggered self-sustaining activity. This activity was confined to a sparse subset of the persistently selective neurons. $\tau_{\mathrm{pg}}$ were longest when the directly photostimulated population was selective for rightward licking (Fig. $1 \mathrm{j}$, Pearson correlation $\left.=0.28, p=5 \times 10^{-5}\right)$. These data show that photostimulation of small groups of neurons can change activity in sparse populations of coupled neurons, and these changes outlast the photostimulus for several seconds.

a
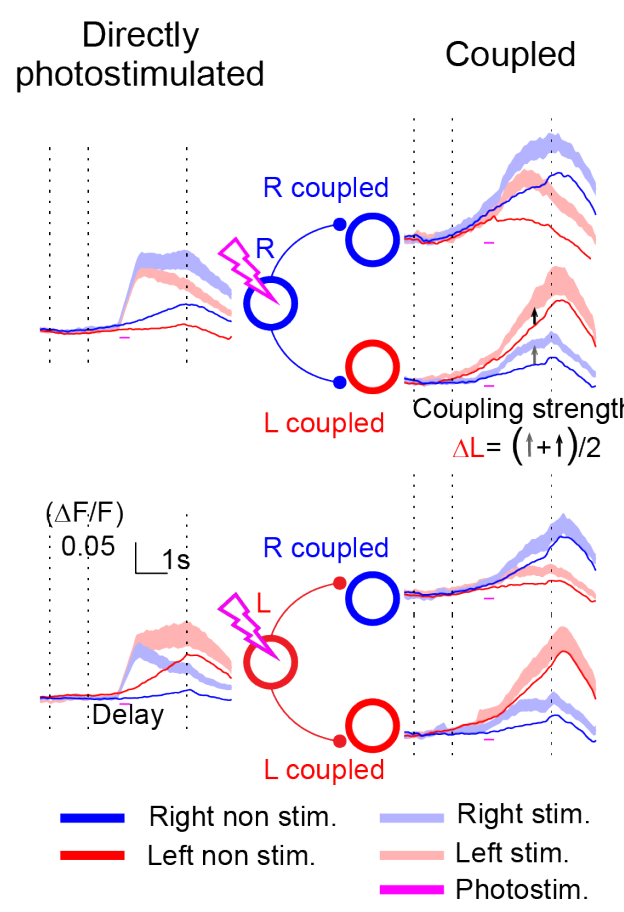

$\mathrm{b}$

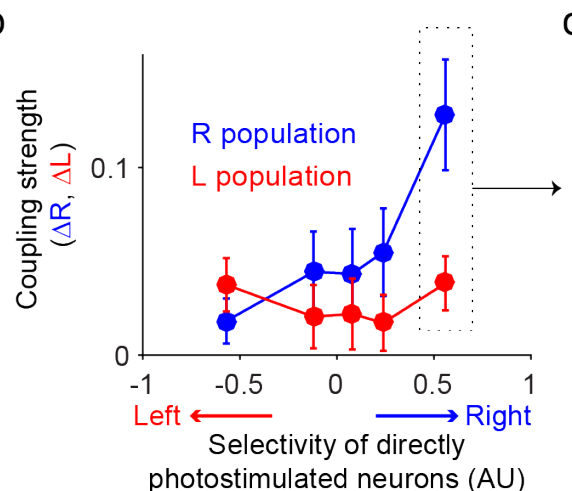

d

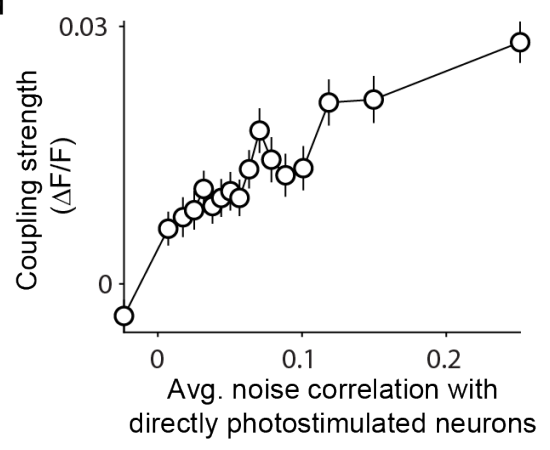

C

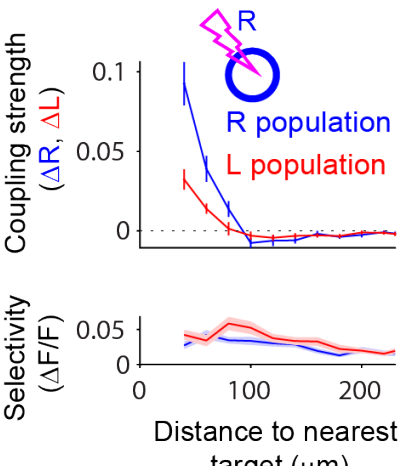
target $(\mu \mathrm{m})$

Figure 2: Functional connectivity depends on response type. a. Analysis of functional connectivity. Left, activity averaged across leftselective ( $L$, red circle) and right-selective ( $R$, blue circle) photostimulation groups (70 each; correct trials only). Dashed vertical lines denote the sample, delay and response epochs. Right, activity of coupled R or L populations. Traces, average activity of all coupled rightor left-selective neurons ( $30-100 \mu \mathrm{m}$ from nearest target) weighted by the strength of their selectivity ( $\mathrm{S}_{\mathrm{i}}^{\text {norm }}$, Methods). Arrows, calculation of coupling strength onto the $L$ population ( $\Delta \mathrm{L}$, Methods); calculation of $\Delta R$ is similar and based on $R$ coupled neurons. $\mathbf{b}$. Coupling strength $(\Delta R$ and $\Delta \mathrm{L}$ ) vs. average selectivity of directly photostimulated neurons (Fig. 1f). Data were binned in quintiles along $x-$ axis (Error bars, s.e.m.). c. Top, Coupling strength ( $\Delta \mathrm{R}$ and $\Delta \mathrm{L}$ ) in response to photostimulation of $\mathrm{R}$ neurons vs. distance to nearest photostimulation target (corresponding to box in b.). Bottom, average selectivity of coupled $R$ and $L$ populations vs. distance to nearest photostimulation target. d. Coupling strength in each coupled neuron vs. its average correlation with all directly photostimulated

71 Persistent activity is thought to be generated by recurrent connections between neurons with similar

72 tuning ${ }^{18,19}$. We tested for specificity in functional connectivity by analyzing the responses of coupled

73 neurons. We first analyzed photostimulated and coupled neurons based on their selectivity in trials 
without photostimulation. We determined whether the directly photostimulated population was mostly right-selective (R) (Fig. 2a, top) or left-selective (L) (Fig. 2a, bottom) and then analyzed (Fig. 2a, right) separately the responses of coupled $R$ and $L$ neurons. Responses were stronger in coupled $R$ neurons when R neurons were photostimulated, consistent with like-to-like excitation (Fig. 2b, blue line; Pearson correlation $=0.15, p=0.0025)$. However, coupled $L$ neurons responded weakly to photostimulation of both $R$ and $L$ neurons (Fig. $2 b$, red line; $p=0.37$ ). The like-to-like excitation from $R$ neurons decreased steeply with distance (Fig. 2c, top), even though selective neurons were present throughout the field of view (Fig. 2c, bottom). These results suggest that recurrent excitation between sparse subsets of nearby R neurons (i.e. selective for contralateral movements) may contribute to maintenance of persistent activity. In addition, the strength of coupling was largest in neurons with trial-to-trial variability that was most correlated with the directly photostimulated neuronal population (Fig. $2 \mathrm{~d}$; Pearson correlation = $0.05, p<10^{-5}$ ), suggesting that coupling reflected synaptic interactions, and consistent with like-to-like excitation.

Selective delay epoch activity of ALM neural populations is causally linked to the direction of future licking $^{12}$. Because targeted photostimuli produced changes in delay epoch activity that persisted until movement onset we tested for effects on animal behavior. A substantial proportion of photostimulation groups produced changes in behavior ( $p<0.05$, bootstrap; $22 / 215$ and $35 / 215$ photostimulation groups on right and left trials respectively) (Fig. 3a), nearly two-fold greater than expected by chance (Fig. 3b, Extended Data Fig. 8, $p<0.001$ Kolmogorov-Smirnov test; Methods). These behavioral changes are consistent with other studies that have manipulated small numbers of neurons during behavior ${ }^{20-22}$.

94 To test how behavioral changes relate to selectivity of the photostimulation group, we calculated the net selectivity produced by the perturbation at the population level. For each imaged neuron we separately calculated the average photostimulated change in activity and the average selectivity. We then defined the 'activated population selectivity' as the product of these two quantities, summed across the 
bioRxiv preprint doi: https://doi.org/10.1101/623785; this version posted April 30, 2019. The copyright holder for this preprint (which was not certified by peer review) is the author/funder. All rights reserved. No reuse allowed without permission. and behavior.

a
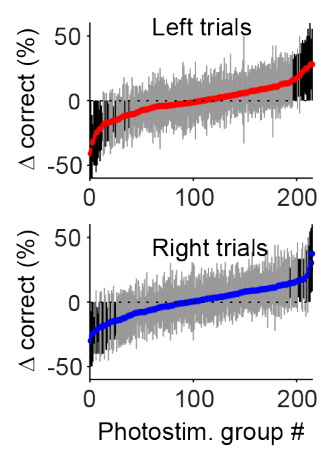

d

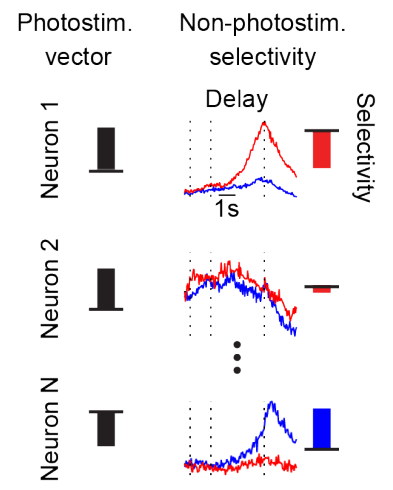

b

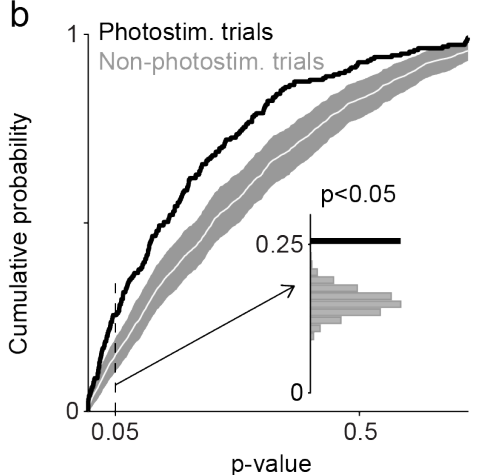

e

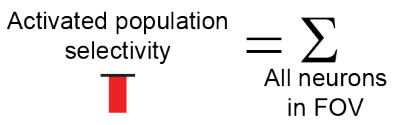

Non-photostim.
selectivity

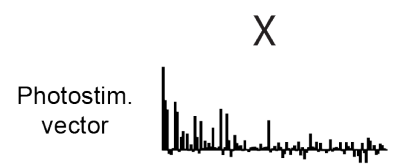

C

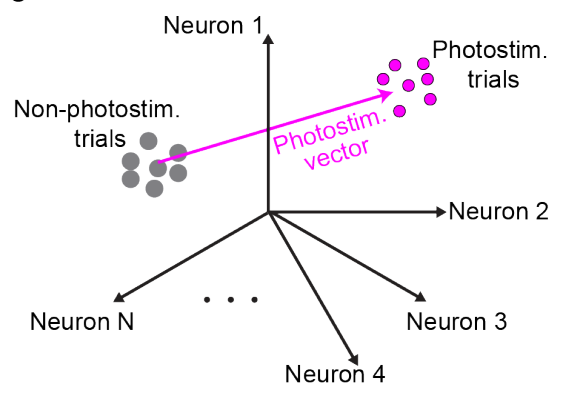

$f$

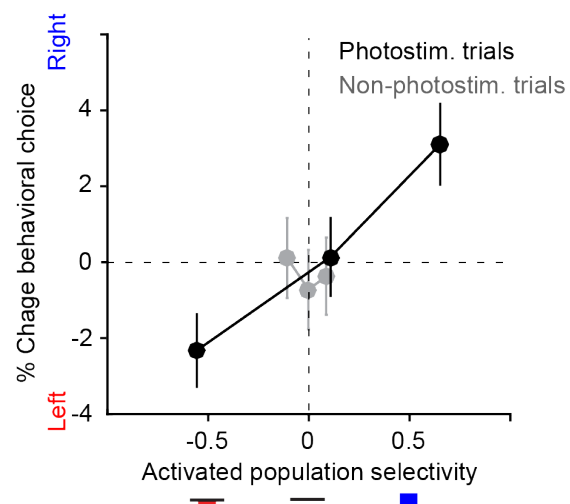

Figure 3: Photostimulation causes predictable changes in behavior. a. Change in behavioral performance between photostimulation and non-photostimulation trials for each photostimulation group for left (top) and right (bottom) trials. Bars represent $95 \%$ confidence intervals of the bootstrap. Black bars, $p<0.05$. b. Cumulative distribution of $p$-values from individual sessions (bars from a) for photostimulation (black) and non-photostimulation (white) trials. Gray bar represents $95 \%$ confidence interval of bootstrap. Inset: Distribution of $\mathrm{p}<0.05$ for photostimulation (black) and non-photostimulation trials (gray). $\mathrm{c}$. Schematic, photostimulated change in neural population in activity space. To take into account changes caused by photostimulation compared to trial-to-trial changes we computed the photostimulation vector (Methods). d. Left, photostimulation vector, contributions of individual neurons. Right, selectivity of individual neurons ( $\mathrm{S}_{\mathrm{i}}$, Methods). e. Activated population selectivity is the dot product of selectivity $\left(\mathrm{S}_{\mathrm{i}}\right)$ and the photostimulation vector. $\mathrm{f}$. Change in percentage of right licking vs. activated population selectivity on photostimulation trials (Errorbars, s.e.m.). network connectivity underlying neural computation ${ }^{8}$. Targeted photostimulation places additional 

persistent changes in activity (Fig. 4a, Extended Data Fig. 10). network as a whole produced sparse persistent activity consistent with experiments.

a
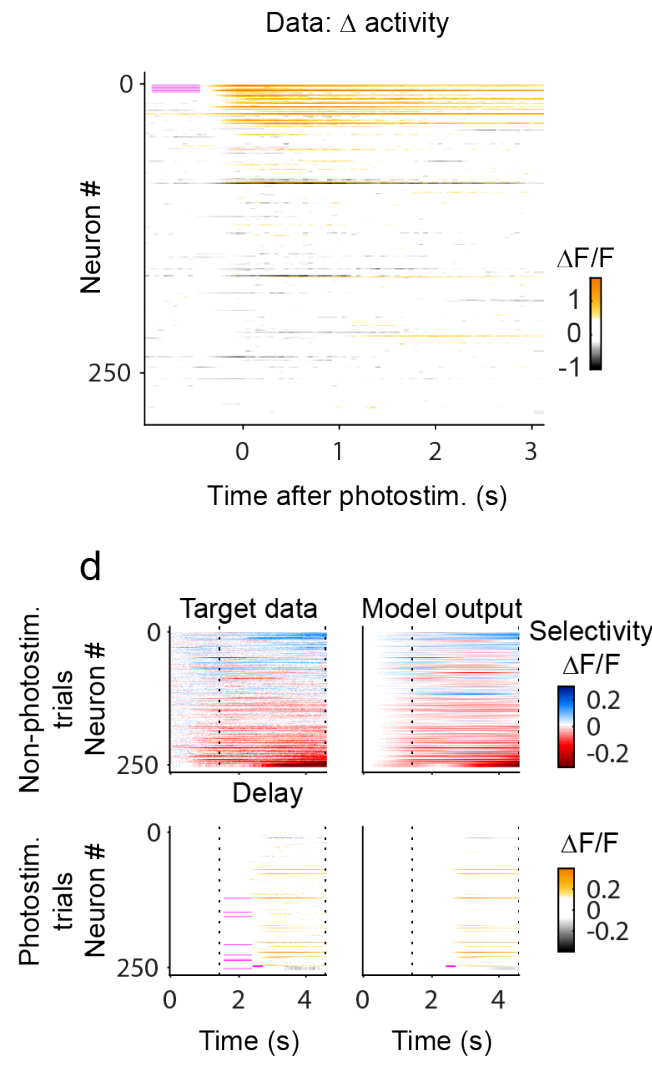

b c

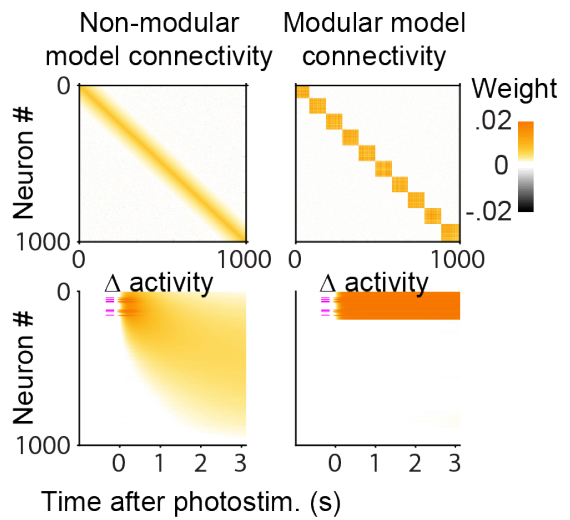

e

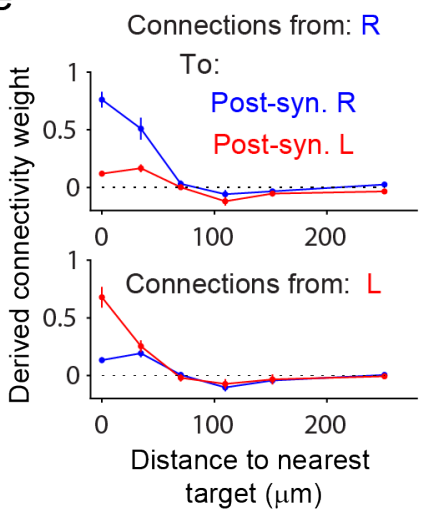

Figure 4: Modular network architecture explains sparse and persistent photostimulation responses. a. $\Delta$ activity of recorded neurons in one experimental session to one photostimulation group ( 8 neurons; magenta lines). b-c. Connectivity (top) and response to targeted photostimulation (bottom; magenta bars, photostimulated neurons) of model networks with locally biased connectivity with non-modular (b) and modular organization (c). Model neurons were sorted according to spatial location. d. Data-driven inference of connectivity. Model connections were tuned to match the model output (right panels) to the recorded data (left panels). Dashed lines denote the sample and delay epochs. e. Connection weights from presynaptic $L$ (bottom panel) and $R$ (top panel) neurons onto post-synaptic $R$ (blue) and $L$ (red) neurons as a function of distance between neurons. 
112 To further test if this modular architecture is consistent with targeted photostimulation experiments, we

$113 \mathrm{fit}^{25}$ model networks directly to the population recordings in photostimulated and non-photostimulated

114 trials (Fig. 4d). The resultant inferred networks were biased towards local connectivity (Fig. 4e), reflecting

115 the length scale of the photostimulation-response (Fig. 1g, Fig. 2c) and local cortical connectivity ${ }^{26,27}$.

116 Connections between R neurons were stronger than connections between L neurons (Fig. 4e) enabling

117 the inferred networks to produce longer time scale responses to photostimulation of R neurons than $\mathrm{L}$

118 neurons (Fig. 1j). Analysis of the local connectivity revealed that the inferred networks resemble the

119 modular network in Figure 4c (Extended data Fig. 10). Networks trained to match only the non-

120 photostimulation trials exhibited significantly weaker connections that resembled the monolithic network

121 (Fig. 4b), demonstrating the power of perturbation experiments to constrain neural network models.

122 We used calcium imaging simultaneously with targeted photostimulation to probe coupling of functionally

123 identified groups of neurons during behavior. These experiments revealed "like-to-like" connectivity

124 between R neurons in the left hemisphere of ALM, and less tuned excitation for L neurons, consistent with

125 previous experiments showing stronger noise correlations in spiking between $R$ neurons than $L$ neurons ${ }^{15}$.

126 Transient perturbations of small groups of neurons caused network responses lasting several seconds,

127 modifying the internal state of a key-circuit for decision-making and modulating behavior seconds later.

128 Even when considering neurons with similar functional properties in a neighborhood, the evoked

129 persistent responses were limited to a subset of neurons. In standard attractor models, activation would

130 spread globally. We propose modular networks with dynamics that are consistent with the data. We

131 hypothesize that these networks may be less sensitive to noise than line-attractor networks, and have

132 higher memory capacity than discrete attractor networks.

133 Our finding that local photostimulation produces persistent changes can be reconciled with previous 134 studies showing that ALM dynamics recover rapidly from network-wide photoinhibition ${ }^{18,19}$. For example, 135 the shapes of within-module energy troughs could have a flat bottom; strong, network-wide perturbations 
136 could selectively engage inter-modular dynamics or non-linear interactions with different circuits (e.g.

137 thalamocortical interactions ${ }^{28}$; Extended data Fig. 10). The wide range of length scales over which

138 interactions produce robust, attractor-like dynamics in our models motivate future experiments in which

139 larger numbers of neurons can be photostimulated ${ }^{29,30}$ over varying length scales. 
140 Acknowledgements:

141 We thank R. Darshan, B. Mohar, A. Finkelstein, H. Inagaki, S. Romani, A. Singh, M. Pachitariu and S. Peron

142 for comments on the manuscript; T. Pluntke and R. Mohar for animal training; X. Zhang, K. Ritola and H.

143 Inagaki for making the ST-chrimsonR constructs; E. Fardone for histology; and P. Rickgauer for discussions.

144 This work was funded by Howard Hughes Medical Institute. KD is a Helen Hay Whitney Foundation 145 postdoctoral fellow and was supported by the Simons Collaboration on the Global Brain. 


\section{References}

147 1. Funahashi, S., Bruce, C. J. \& Goldman-Rakic, P. S. Mnemonic coding of visual space in the monkey's dorsolateral prefrontal cortex. J. Neurophysiol. 61, 331-349 (1989).

2. Romo, R., Brody, C. D., Hernández, A. \& Lemus, L. Neuronal correlates of parametric working memory in the prefrontal cortex. Nature 399, 470-473 (1999).

3. Goldman, M. S. Memory without Feedback in a Neural Network. Neuron 61, 621-634 (2009).

4. Lim, S. \& Goldman, M. S. Balanced cortical microcircuitry for maintaining information in working memory. Nat Neurosci 16, 1306-1314 (2013).

5. Cannon, S. C., Robinson, D. A. \& Shamma, S. A proposed neural network for the integrator of the oculomotor system. Biol Cybern 49, 127-136 (1983).

6. Chaudhuri, R. \& Fiete, I. Computational principles of memory. Nature Neuroscience 19, 394-403 (2016).

7. Wang, X.-J. Synaptic reverberation underlying mnemonic persistent activity. Trends in Neurosciences 24, 455-463 (2001).

8. Denk, W., Briggman, K. L. \& Helmstaedter, M. Structural neurobiology: missing link to a mechanistic understanding of neural computation. Nature Reviews Neuroscience 13, 351-358 (2012).

9. Rickgauer, J. P., Deisseroth, K. \& Tank, D. W. Simultaneous cellular-resolution optical perturbation and imaging of place cell firing fields. Nat Neurosci 17, 1816-1824 (2014). and recording of neural circuit activity with cellular resolution in vivo. Nat Meth 12, 140-146 (2015). (2014). 300 (2013). 
13. Klapoetke, N. C. et al. Independent optical excitation of distinct neural populations. Nat Meth 11, 338-346 (2014).

14. Chettih, S. N. \& Harvey, C. D. Single-neuron perturbations reveal feature-specific competition in V1. Nature 567, 334 (2019).

15. Inagaki, H. K., Inagaki, M., Romani, S. \& Svoboda, K. Low-Dimensional and Monotonic Preparatory Activity in Mouse Anterior Lateral Motor Cortex. J. Neurosci. 38, 4163-4185 (2018).

16. Chen, T.-W., Li, N., Daie, K. \& Svoboda, K. A Map of Anticipatory Activity in Mouse Motor Cortex. Neuron 94, 866-879.e4 (2017).

17. Li, N., Chen, T.-W., Guo, Z. V., Gerfen, C. R. \& Svoboda, K. A motor cortex circuit for motor planning and movement. Nature 519, 51-56 (2015).

18. Li, N., Daie, K., Svoboda, K. \& Druckmann, S. Robust neuronal dynamics in premotor cortex during motor planning. Nature 532, 459-464 (2016).

19. Inagaki, H. K., Fontolan, L., Romani, S. \& Svoboda, K. Discrete attractor dynamics underlies persistent activity in the frontal cortex. Nature 566, 212 (2019).

20. Tanke, N., Borst, J. G. G. \& Houweling, A. R. Single-Cell Stimulation in Barrel Cortex Influences Psychophysical Detection Performance. J. Neurosci. 38, 2057-2068 (2018).

21. Huber, D. et al. Sparse optical microstimulation in barrel cortex drives learned behaviour in freely moving mice. Nature 451, 61-64 (2008).

22. Jennings, J. H. et al. Interacting neural ensembles in orbitofrontal cortex for social and feeding behaviour. Nature 565, 645-649 (2019).

23. Seung, H. S. How the brain keeps the eyes still | PNAS. Available at: http://www.pnas.org/content/93/23/13339.short. (Accessed: 4th December 2018)

24. Hopfield, J. J. Neural networks and physical systems with emergent collective computational abilities. Proc. Natl. Acad. Sci. U.S.A. 79, 2554-2558 (1982). 
194 25. Rajan, K., Harvey, C. D. \& Tank, D. W. Recurrent Network Models of Sequence Generation and

$195 \quad$ Memory. Neuron 90, 128-142 (2016).

196 26. Holmgren, C., Harkany, T., Svennenfors, B. \& Zilberter, Y. Pyramidal cell communication within local

197 networks in layer 2/3 of rat neocortex. J Physiol 551, 139-153 (2003).

198 27. Komiyama, T. et al. Learning-related fine-scale specificity imaged in motor cortex circuits of

199 behaving mice. Nature 464, 1182-1186 (2010).

200

28. Guo, Z. V. et al. Maintenance of persistent activity in a frontal thalamocortical loop. Nature 545, 181-186 (2017).

202

29. Mardinly, A. R. et al. Precise multimodal optical control of neural ensemble activity. Nature Neuroscience 21, 881 (2018).

30. Yang, W., Carrillo-Reid, L., Bando, Y., Peterka, D. S. \& Yuste, R. Simultaneous two-photon imaging and two-photon optogenetics of cortical circuits in three dimensions. eLife 7, e32671 (2018). 
GCaMP6s

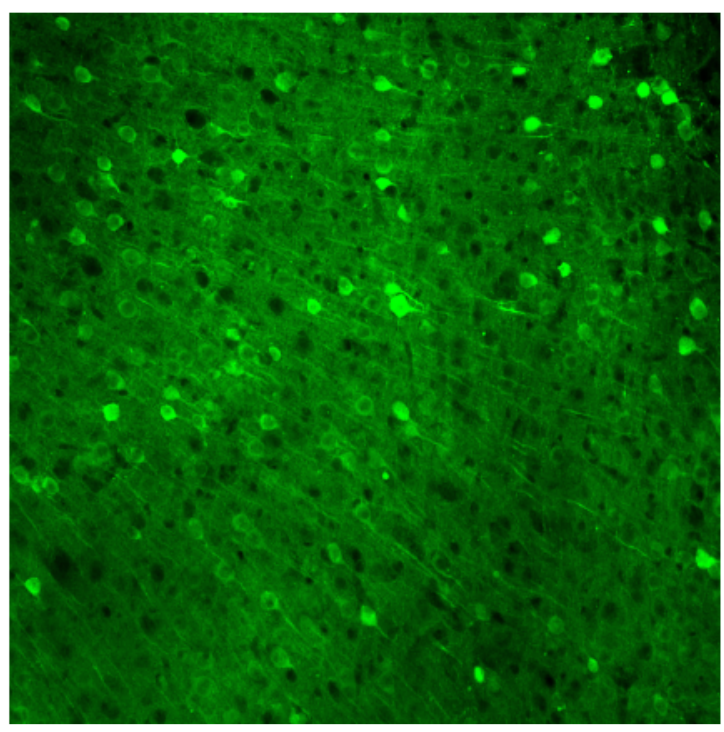

ST-chrimsonR-FusionRed

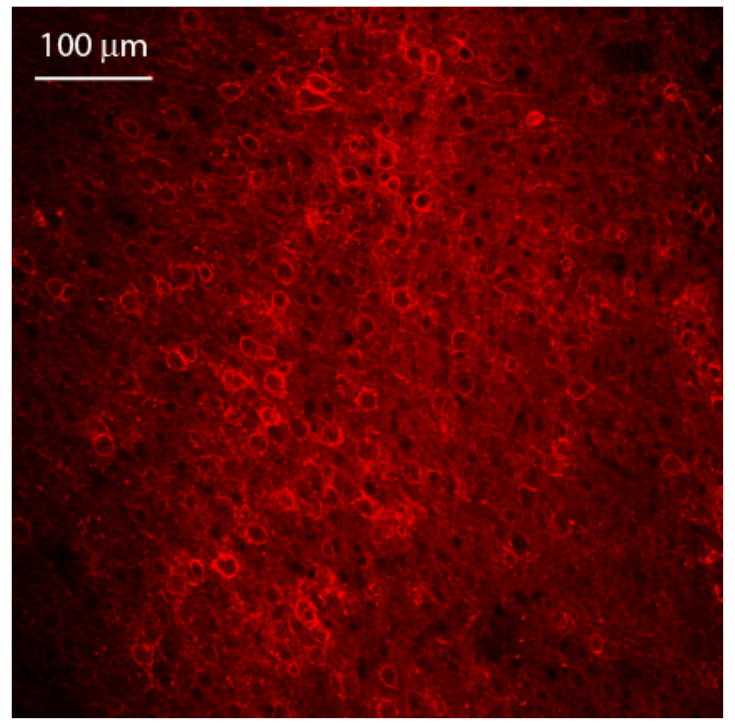

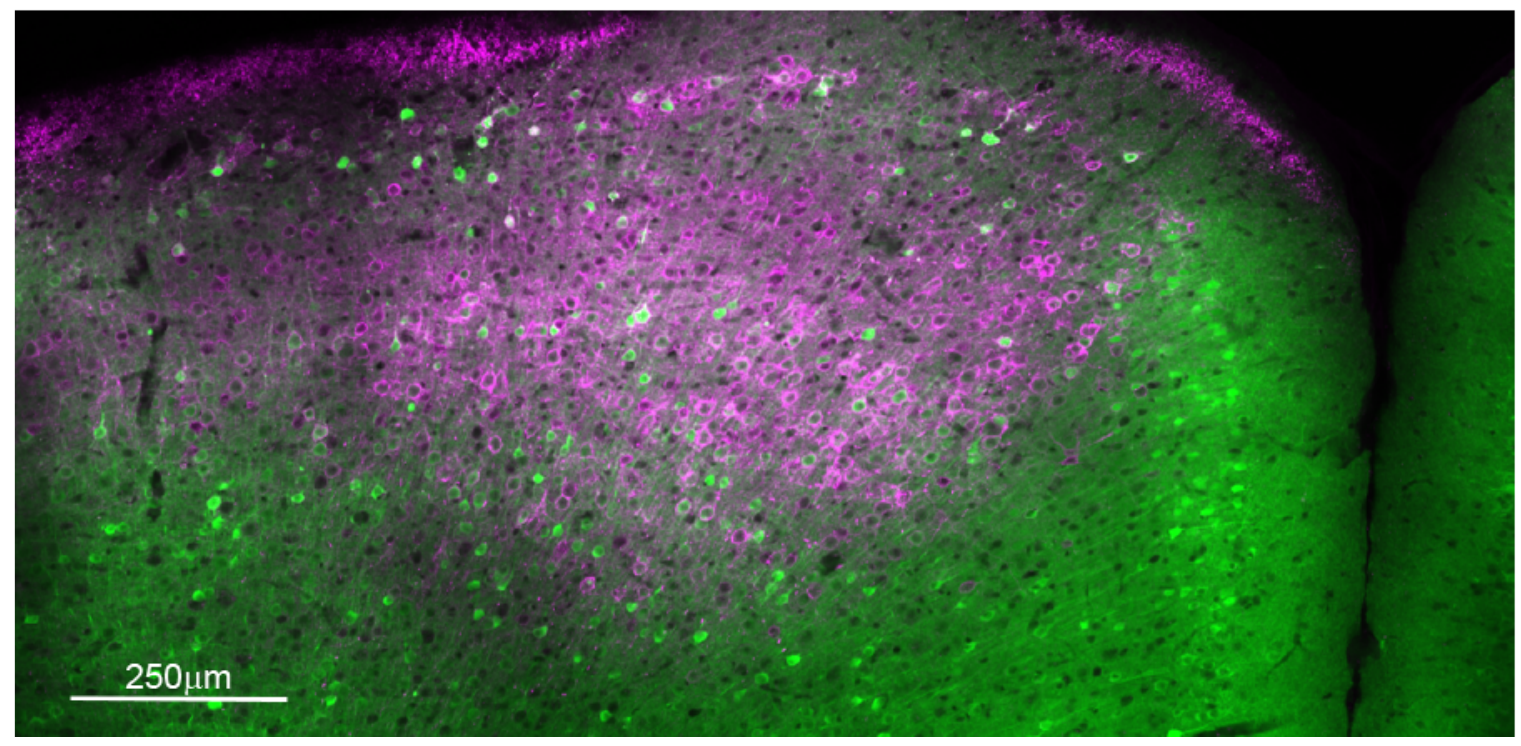

209 Extended Data Figure 1: Coexpression of GCaMP6s and ST-chrimsonR. After experiments brains were

210 harvested and sectioned (100 $\mu \mathrm{m}$ coronal sections). Images show co-expression of GCaMP6s (green) and

211 ST-chrimsonR-FusionRed (red) from a section that was under the cranial window. 
a

Microscope for simultaneous 2-photon imaging and photostimulation

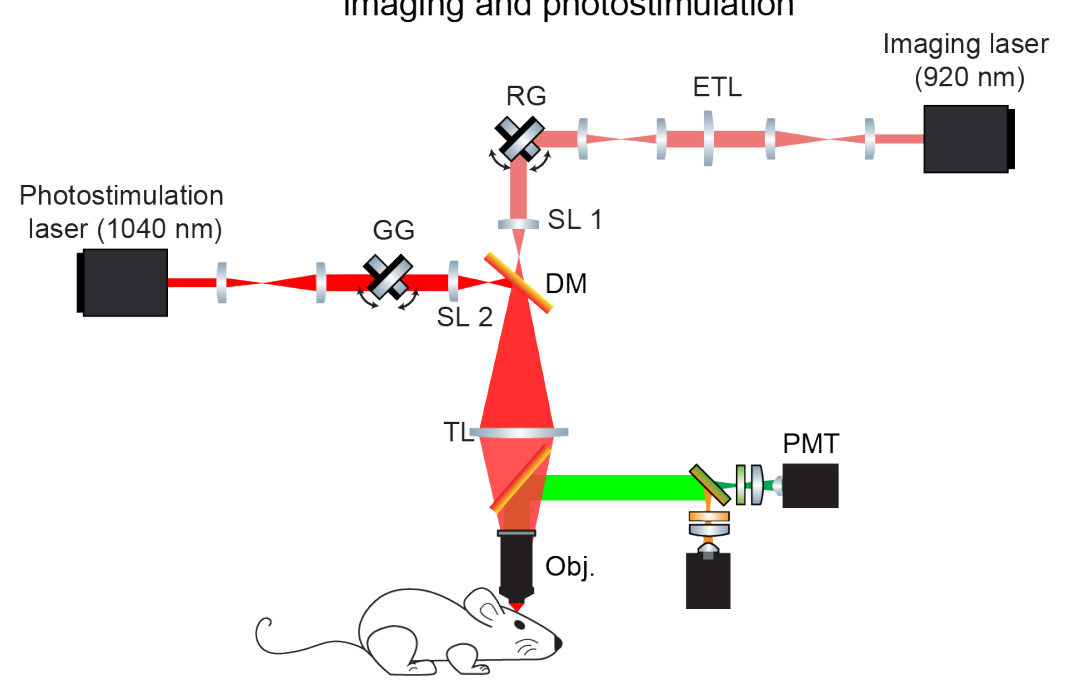

b

Axial resolution

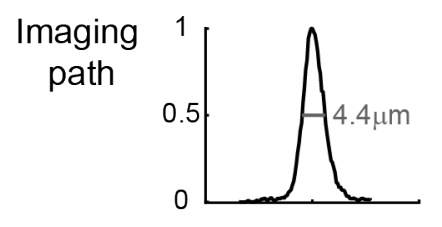

Photostim.

path

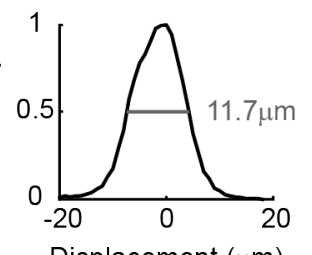

Lateral resolution
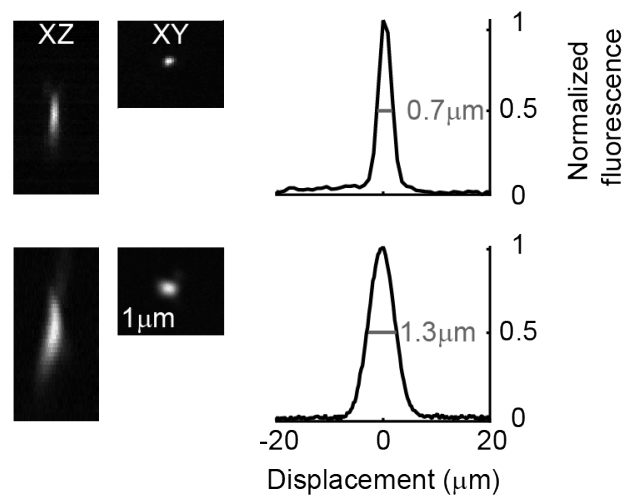

Extended Data Figure 2: Simultaneous imaging and targeted photostimulation. a. Schematic of the microscope: Photostimulation laser, 1040 nm (Fidelity HP, Coherent); Imaging laser, 920 nm (Chameleon

215 Ultra II, Coherent); GG, pair of $3 \mathrm{~mm}$ galvanometer mirrors (Cambridge, 6215H); ETL, electric tunable lens,

216 (EL-10-30-C, Optotune); SL 2, scan lens photostimulation path, $33 \mathrm{~mm}$ focal length, a stack of $3 \times 100 \mathrm{~mm}$

217 focal length lenses (AC-254-100b, Thorlabs); SL 1, scan lens imaging path, $30 \mathrm{~mm}$ focal length (55-S30-

218 16T, Special Optics); DM, $1000 \mathrm{~nm}$ short-pass dichroic mirror (Edmund optics); TL, Tube lens $160 \mathrm{~mm}$ focal

219 length (Special Optics); Obj., 16x objective, $0.8 \mathrm{NA}, 3 \mathrm{~mm}$ working distance (CFI75 LWD, Nikon); PMT,

220 photomultiplier tubes (H10770(P)B-40, Hamamatsu). b. Point-spread functions. Measurements were 
bioRxiv preprint doi: https://doi.org/10.1101/623785; this version posted April 30, 2019. The copyright holder for this preprint (which was not certified by peer review) is the author/funder. All rights reserved. No reuse allowed without permission.

221 made by imaging $500 \mathrm{~nm}$ yellow-green beads (Polysciences). Reported values correspond to the full-width 222 at half maximum. 
a

Selectivity $(\Delta F / F)$

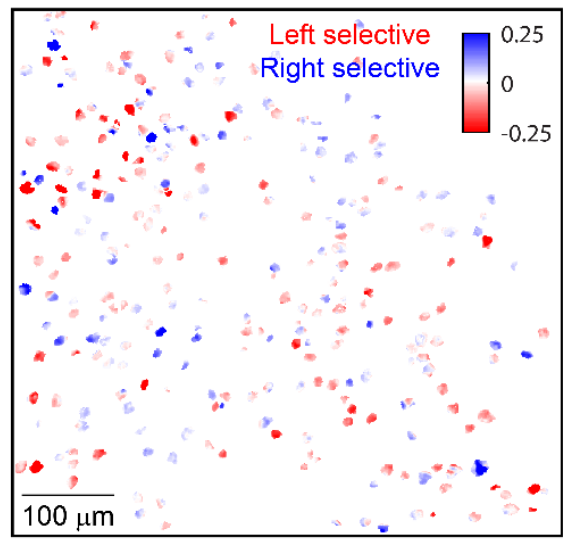

b

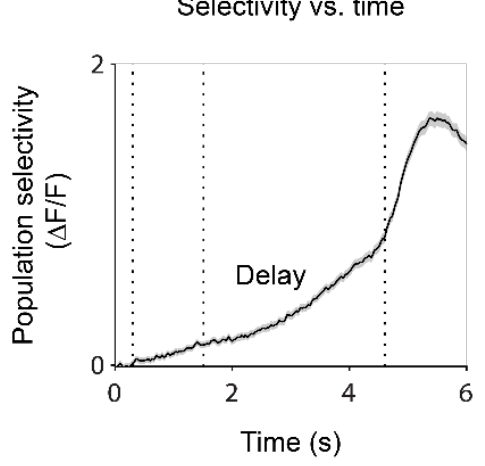

Left selective neurons

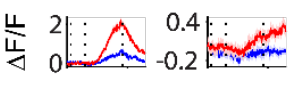

${ }_{0}^{1.5}: \begin{array}{rr}0.4 \\ 0\end{array}$
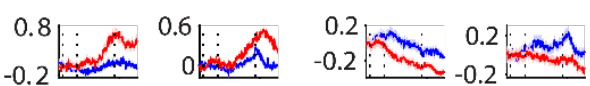

$0.8: \vdots{ }_{-0.2}^{0.4}:-4$
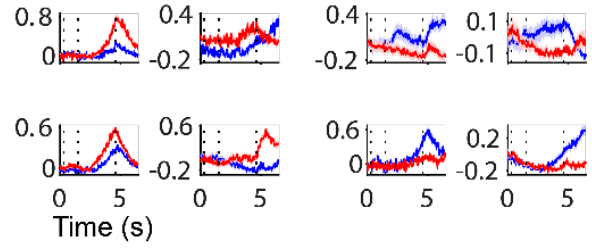

C

$$
\text { Number of selective neurons }
$$

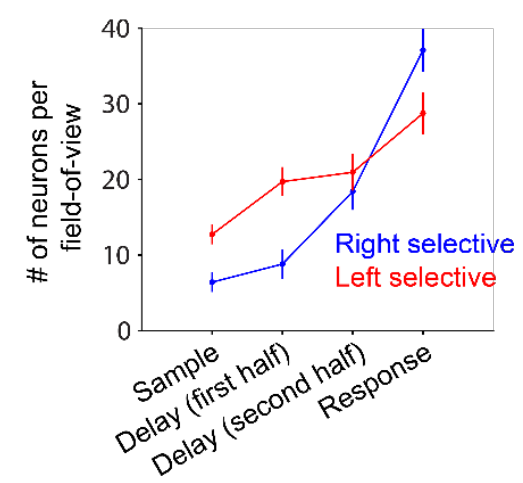

Extended Data Figure 3: Trial-type selectivity. a. Map of selectivity for individual neurons in one

227 the end of the delay epoch. Selective neurons were scattered across the field of view (left panel), and

228 displayed heterogenous dynamics (right panel, example neurons). Dashed vertical lines denote the

229 sample, delay and response epochs.

b. For each behavioral session all neurons were categorized based on their selectivity at each time point:

$$
V_{i}^{\text {sel. } .}(t)=\operatorname{Sign}\left(\left\langle f_{i, j}^{R, \text { non }}(t)\right\rangle_{j=(\text { correct trials })_{1}}-\left\langle f_{i, j}^{L, \text { non }}(t)\right\rangle_{j=\text { correct trials }}\right)
$$


232 where trial averaging was performed over a randomly-chosen training subset of $20 \%$ of correct trials

233 denoted as "correct trials". With the remaining $80 \%$ of trials (correct trials $s_{2}$, testing subset) we computed

234 the population selectivity as:

235 Population selectivity $(t)=1 / \sqrt{(N)} \sum_{i=1 \ldots N}\left(\left\langle f_{i, j}^{R, \text { non. }}(t)\right\rangle_{j=\text { correct trials }_{2}}-\left\langle f_{i, j}^{L, \text { non. }}(t)\right\rangle_{j=\text { correct trials }_{2}}\right) V_{i}^{\text {sel. }}$

236 where $\mathrm{N}$ is the number of neurons in the FOV. Dashed vertical lines indicated the sample, delay and

237 response epochs, Errorshade is the s.e.m. across sessions.

238 c. We used a T-test to compare the epoch-averaged activity on left and right trials for each neuron:

$$
\bar{f}_{i, j}^{R, \text { non. }}=\left\langle f_{i, j}^{R, \text { non. }}(t)\right\rangle_{t=\text { epoch }} \quad \& \bar{f}_{i, j}^{L, n o n .}=\left\langle f_{i, j}^{L, \text { non. }}(t)\right\rangle_{t=\text { epoch }}
$$

240 We then counted the number of neurons for which the resulting $p$-value was less than 0.05 . 
a

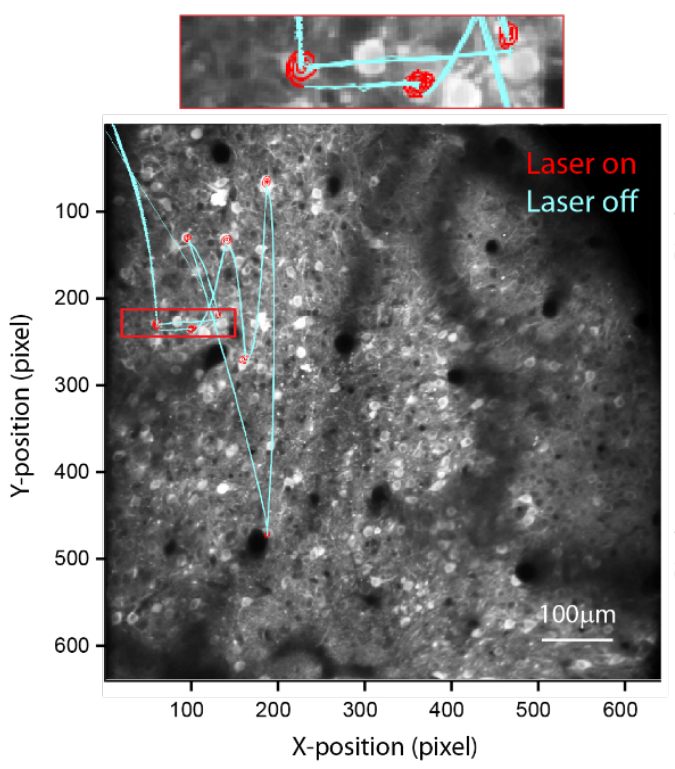

b

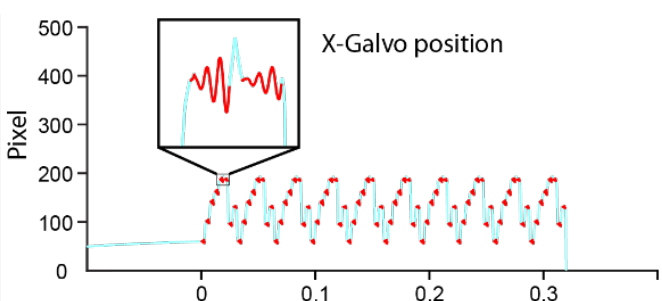

Y-Galvo position

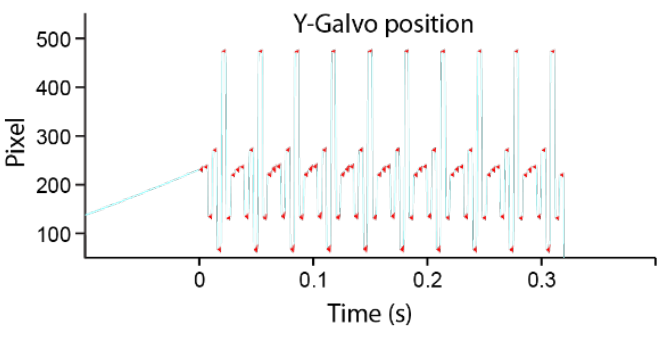

243 Extended Data Figure 4: Photostimulation scan trajectory. a. Photostimulation was performed by

244 scanning the laser over a target neuron, defined by GCaMP6s fluorescence, in a spiral pattern. The image

245 shows the scan path, reconstructed from position encoders of the galvo mirrors, superposed on a

246 fluorescence image. Each spiral (red) lasted for $3 \mathrm{~ms}$. Laser power was then turned off for $1 \mathrm{~ms}$ while the

247 beam was redirected to the next neuron in the sequence (cyan). Once the last ( ${ }^{\text {th }}$ ) neuron was

248 photostimulated the beam was directed back to the first neuron. This sequence was repeated 10 times 249 per photostimulation trial (b). 
a

Spikes evoked in the targeted neuron

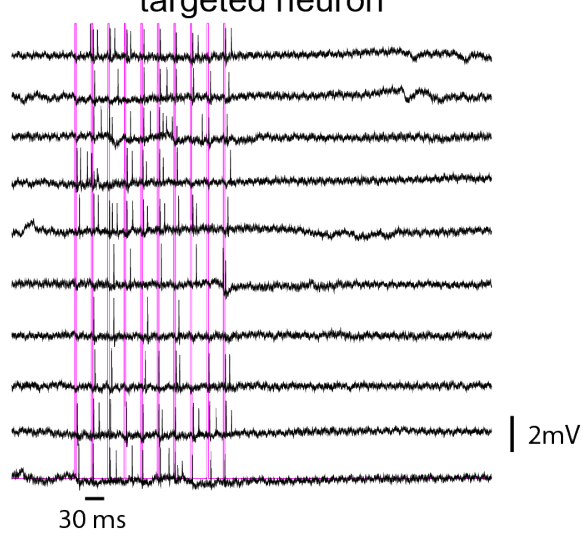

d Electrophysiology and 2-photon calcium imaging
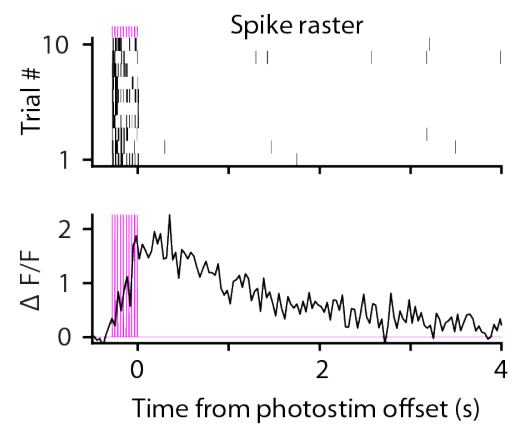

b

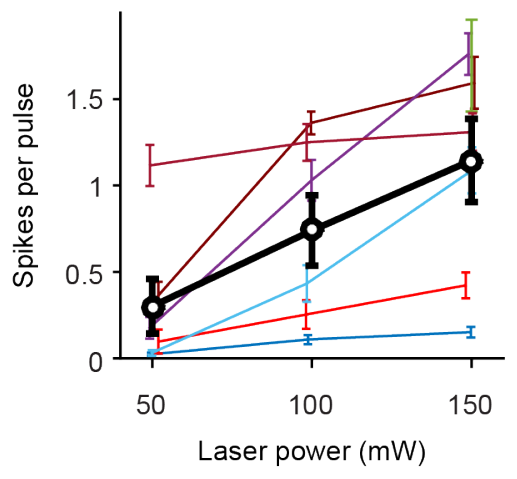

C

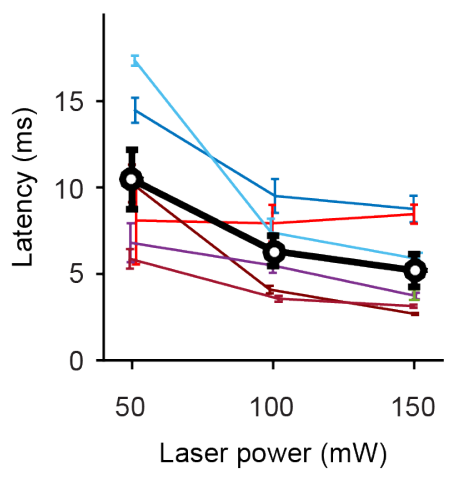

Extended Data Figure 5: Electrophysiology during targeted photostimulation. Loose-seal, cell-attached recordings during photostimulation in anesthetized mice. Single neurons $(n=7)$ were photostimulated using the pattern shown in Extended Data Figure 4a with either 50, 100 or $150 \mathrm{~mW}$ laser power. a. Photostimuli produced increases in spiking. Red bars, photostimuli. b. Spikes evoked by single photostimuli (spiral) as a function of laser power. The weakest photostimuli $(50 \mathrm{~mW})$ failed to drive spikes in most neurons. All photostimulation experiments done during behavior used either 100 or $150 \mathrm{~mW}$, which evoked on the order of one spike per photostimulus. c. Spike latency as a function of laser power. extracellular voltage, showing that photostimulation produced increases in spiking that were associated with large increases in fluorescence. These fluorescence transients decayed with a $1 \mathrm{~s}$ time constant, 
bioRxiv preprint doi: https://doi.org/10.1101/623785; this version posted April 30, 2019. The copyright holder for this preprint (which was not certified by peer review) is the author/funder. All rights reserved. No reuse allowed without permission.

262 consistent with previous observations ${ }^{1}$, and much faster than decays observed when photostimulating 263 groups of neurons during the delay epoch. 


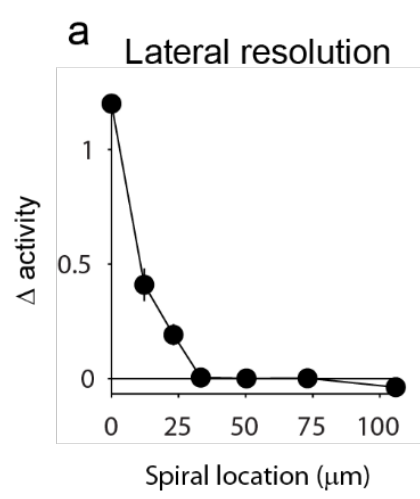

C

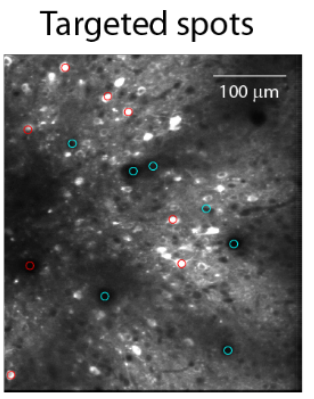

Extended Data Figure 6: Spatial resolution of photostimulation. a. Lateral resolution. Resolution was characterized during quiet wakefulness by measuring the amplitude of photostimulation-induced GCaMP6 transients with the photostimulus at varying distances from the recorded neuron ( $\mathrm{n}=9$ neurons, 10 trials for each photostimulus location). The normalized change in activity was calculated as:
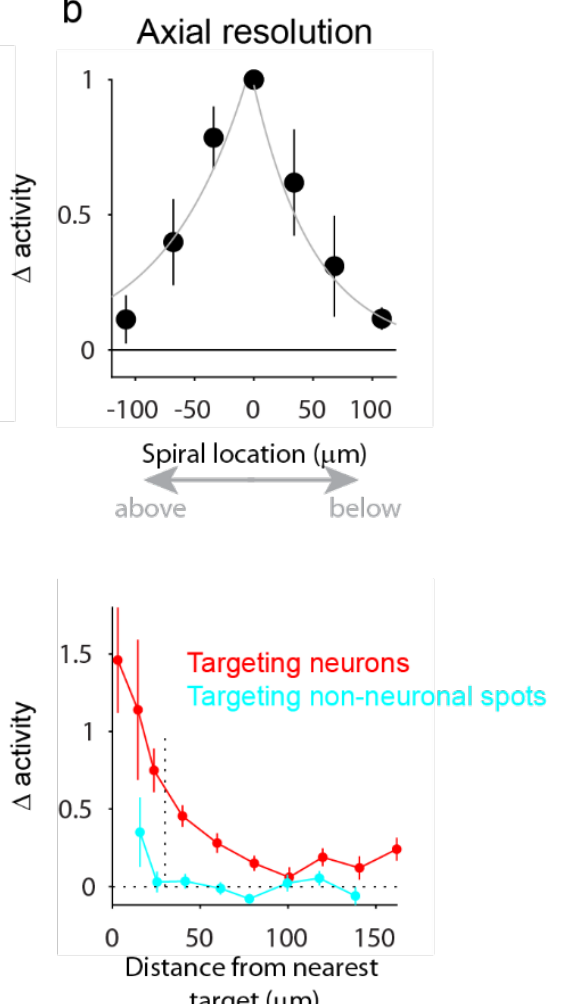
target $(\mu \mathrm{m})$ where $f_{p r e, x y}$ is the average fluorescence before photostimulation $(150 \mathrm{~ms})$ and $f_{\text {post }, x y}$ is the

272 fluorescence averaged immediately after the photostimulation (1 s) at a lateral distance of xy micrometers

273 from the imaged cell. Changes in activity were limited to a radius of less than 30 micrometers. $\mathbf{b}$. The axial

274 resolution in these experiments is 80 micrometers (FWHM), about a factor of 1.5 or 2 larger than reported

275 using similar methods ${ }^{2,3}$. This broadening is because we used stronger photostimuli to produce reliable changes in activity in the targeted neurons. Given variable levels of expression and excitability, these laser 
277 powers were sufficient to drive spikes even in weakly expressing neurons, at the cost of degraded axial

278 resolution ${ }^{4}$. c. Additional resolution measurements in awake mice. Resolution experiments described

279 above were performed during periods of quiet wakefulness. To further account for potential differences

280 in neuronal excitability during behavior we performed additional experiments to estimate the lateral

281 spatial resolution of group photostimulation during behavior. As in Figure 1, we selected groups of 8

282 neurons to photostimulate during behavior (red). Additionally, we selected groups of 8 non-neuronal

283 spots to target for photostimulation (cyan). For the groups of non-neuronal spots, we find that there is

284 weak activation of neurons within 20 microns of a target, but no significant changes were observed more

285 than 30 microns away from a target location. Because photostimulation of non-neuronal spots fails to

286 produce changes in activity at distances greater than 30 microns from the photostimulation target, we

287 treat any neurons located 30 microns or more from a target throughout the text as "coupled". In addition,

288 we treat any neurons located within 20 microns of a target whose activity was significantly altered as

289 "directly photostimulated". 
a

Ephys. \& $2 p$ imaging to estimate GCaMP6s decay (Chen et al, 2016)
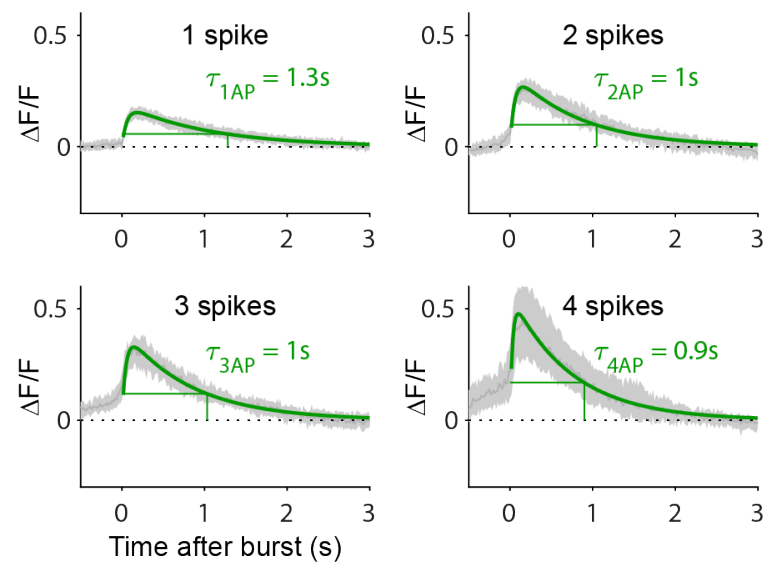

b

Deconvolved fluorescence traces from Figure $1 \mathrm{~h}$

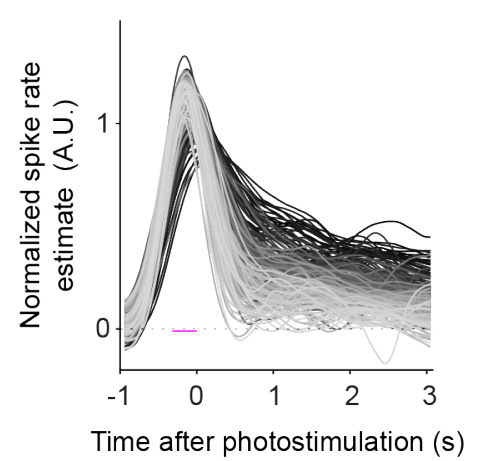

Estimating GCaMP6s decay in ALM during behavior

C Example delay selective neurons (ephys, Li et al., 2016)
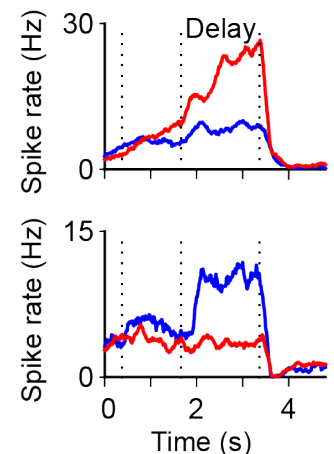

d Avg. of delay selective ALM neurons (GCaMP6s)
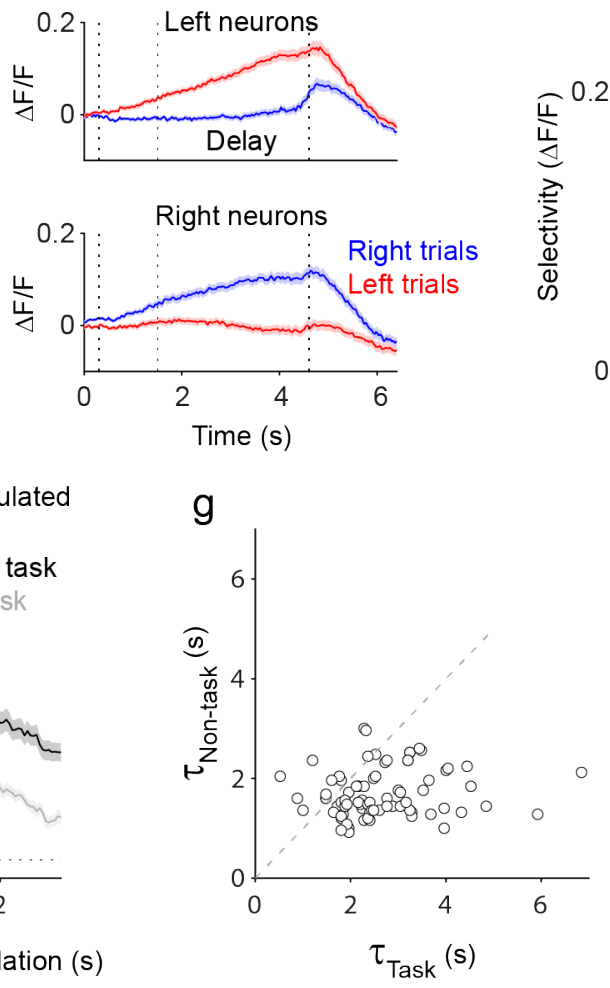

e Avg. selectivity of neurons from d

f

Avg. directly photostimulated

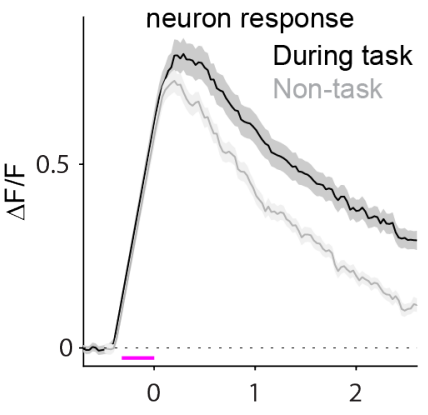

Time after photostimulation (s)

Extended Data Figure 7: Dynamics of activity-dependent fluorescence. a. Estimating GCaMP6s decay after short bursts of activity in anesthetized mice. We analyzed simultaneously recorded cell-attached electrophysiology and two-photon imaging of GCaMP6s in the visual cortex ${ }^{1,5}$ to assess the decay of 
GCaMP6s fluorescence. The decay time constant is the time at which the activity decays to 1/e of its peak value. We analyzed the fluorescence traces following bursts of 1, 2, 3 or 4 action potentials and found $\tau^{\prime}$ s of $1.2 \mathrm{~s}, 1 \mathrm{~s}, 1 \mathrm{~s}$ and $0.9 \mathrm{~s}$ respectively.

b. We fit each fluorescence response with the kernel $\kappa_{G C a M P 6 s}$ (green traces) according to the equation:

$$
\kappa_{G C a M P 6 s}=a e^{-t / \tau_{\text {deay }}}\left(1-e^{-t / \tau_{\text {rise }}}\right)
$$

and obtained the following average parameter values: $\mathrm{a}=0.31 \tau_{\text {decay }}=0.87 \mathrm{~s}$ and $\tau_{\text {rise }}=0.06 \mathrm{~s}$.

We used $\kappa_{\text {GCaMP6s }}$ to estimate the spiking dynamics underlying the fluorescence responses of directly

photostimulated neurons by deconvolving each trace in Figure $1 \mathrm{~h}$ with the kernel $\kappa_{G C a M P 6 s}$. Prior to deconvolution, traces were smoothed with a cubic smoothing spline (MATLAB; csaps, $P=0.96$ ). We found that $95 \%$ of photostimulation groups produce changes in estimated spike rate that remain elevated 2 seconds or longer following photostimulation. c. We also used known rapid spike rate changes in ALM to estimate the GCaMP6s decay time during behavior ${ }^{6}$. ALM contains neurons in which selectivity peaks at the end of the delay epoch and rapidly decays to zero following the response cue. Example neurons with late-delay right (top) and left (bottom) selectivity ${ }^{7}$ are shown. Fluorescence dynamics in these neurons will enable us to estimate the GCaMP6s decay time constant by observing the decay of selectivity following the go-cue. d. We identified neurons with late delay epoch selectivity (Two-tailed T-test, $p<0.2$ ) that was greater than the response response epoch selectivity (Two-tailed T-test, $p>0.2$ ). On average we

312 found 13.5 such neurons per session $(6-22)$. The traces show the average of right (top) and left (bottom) selective neurons. e. We next plotted the average selectivity of these delay selective neurons as calculated

314 in Extended Data Fig. 3. From the electrophysiological recordings, we assume that spike rate selectivity of 315 these neurons drops to zero. We found that selective fluorescence decayed with $\tau=0.98 \mathrm{~s}$, consistent 316 with the measured decay times of GCaMP6s in a. This is expected to be an upper bound on the GCaMP6s 
317 fluorescence decay time because selectivity in some of these neurons may not instantaneously drop to

318 zero and produce erroneously long estimates of $\tau$.

319 f. To test if the persistent changes in activity could also be evoked during periods of quiet wakefulness, on

320 a subset of experiments we photostimulated the same groups of neurons immediately after the animal

321 completed the behavioral session ( 3 mice, 10 photostimulation groups). We found that the activity

322 averaged across all groups and directly photostimulated neurons decayed more slowly during the task

323 than in the non-task period. g. For each directly photostimulated neuron (84 neurons), we calculated the

324 decay time constant both during the task and during the non-task period. Decay time constants during

325 the task $(\tau=3.1 \mathrm{~s}, 1.8 \mathrm{~s}-4.3 \mathrm{~s}, 75 \% \mathrm{Cl})$ were significantly longer than time constants outside of the task

326 ( $\tau=1.7 \mathrm{~s}, 1.2-2.4,75 \% \mathrm{Cl} ; \mathrm{p}<10^{-5}$, two-tailed T-test; errorbars, s.e.m.). 
a

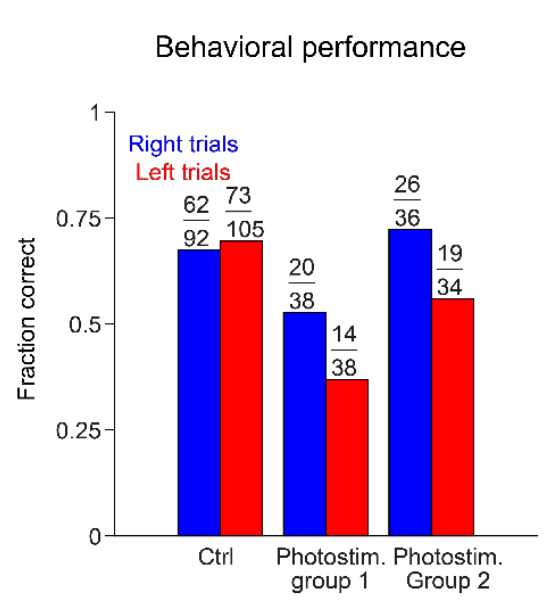

328

329

330

331

333

334

335

336

337

338

339

340 triggered only sparse changes in activity. b

Expected number of correct trials

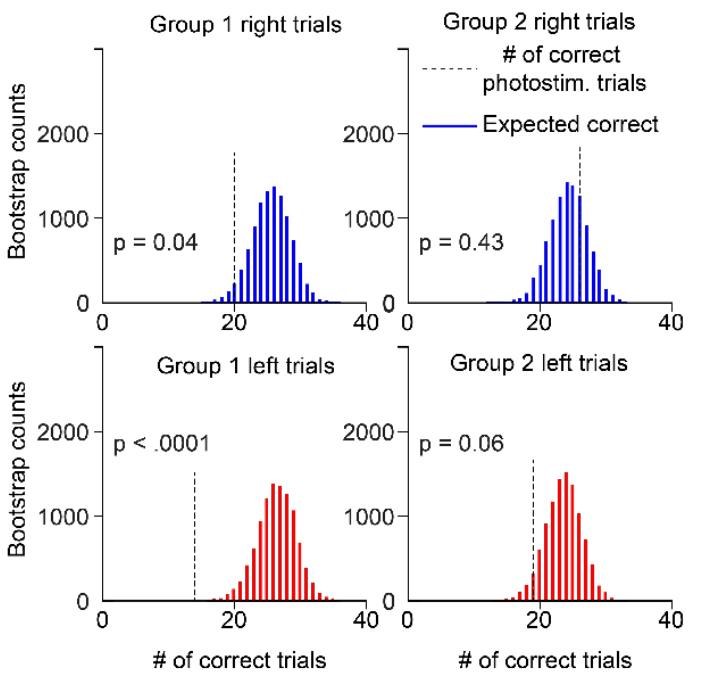

C

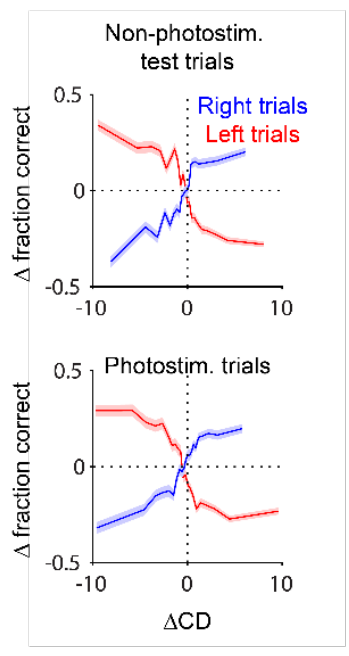

Extended Data Figure 8: Effect of photostimulation on behavior. a. Behavioral performance of a mouse on a single session. Performance dropped from 73/105 correct (69\%) on non-photostimulation lick left trials to $14 / 38$ (37\%) when photostimulation group 1 was photostimulated. b. A bootstrap distribution of performance on non-photostimulation trials was generated by randomly sampling 38 of the 105 nonphotostimulation trials 10,000 times (b, bottom left). For each of the 10,000 random samplings, we counted the number of correct trials to determine the $p$-value for each photostimulation group. Bootstrap distributions were used to determine the $95 \%$ confidence intervals in Figure $3 a$.

c. To relate changes in performance to changes in activity, we projected population activity along a "coding direction" (CD) which maximally separates trial-averaged activity on a subset of "training" left and right non-photostimulation trials ${ }^{8}$. We then projected single-trial activity from a testing subset of nonphotostimulation trials (top) as well as photostimulation trials (bottom) along the CD and calculate the average change in performance vs. displacement along the $C D$. The relationship between $C D$ activity and behavior was similar for photostimulation and non-photostimulation trials because photostimulation 
a
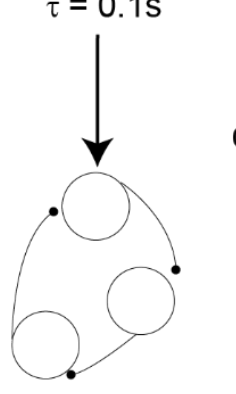

$\tau=1 \mathrm{~s}$

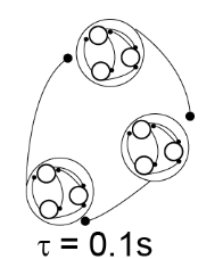

b

C
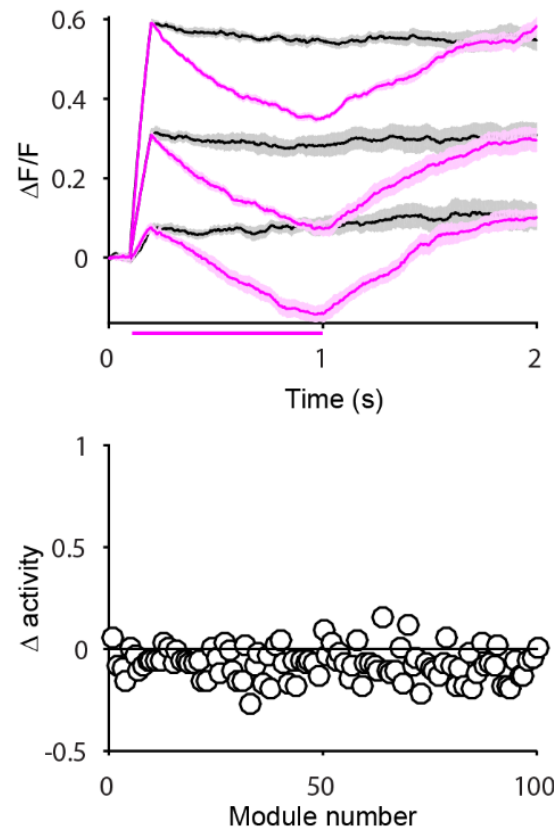

$\mathrm{h}$

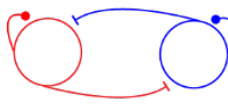

g

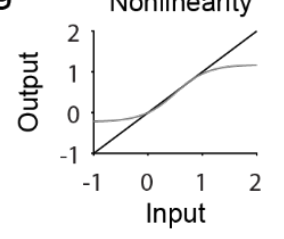

Broad photoinhibition

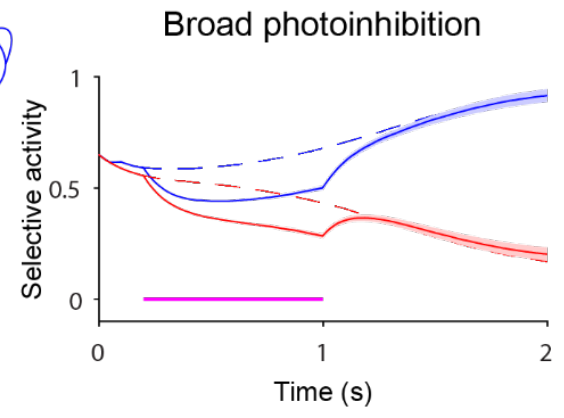

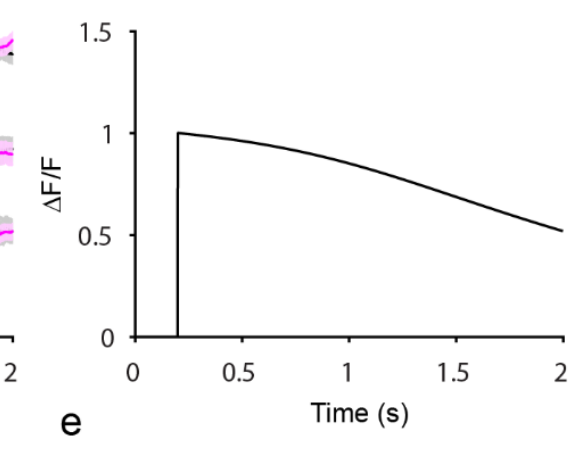

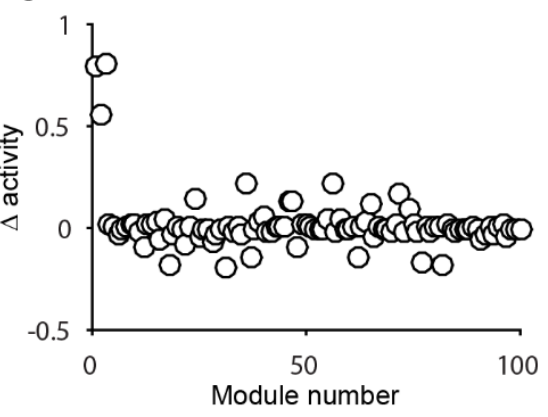

i

Targeted photostimulation

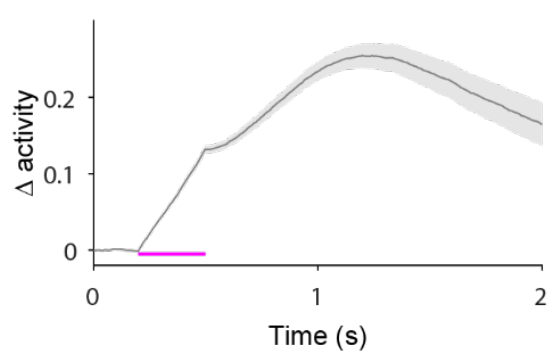

Targeted photostimulation

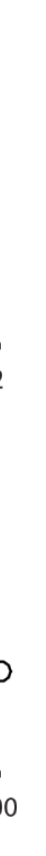

d

345 Extended Data Figure 9. Network models of robustness. The model in Figure 4d-e produces persistent

346 selectivity and sparse persistent responses to photostimulation through a locally connected modular

347 architecture. The model was fit directly to the data, and all of the relevant connections for maintaining

348 persistence are between a small number of neighboring neurons. The toy model network in Figure 4c

349 illustrates this modularity. This model produces sparse persistent responses to photostimulation of a small

350 number of neurons. However, this model does not by itself produce the rapid recovery from broad

351 photoinhibition that has been described in $\mathrm{ALM}^{9}$. 
One possible reconciliation of these two findings can be achieved by tuning intermodular connections to

353

produce rapid recovery from broad photoinhibition. a. We made a simplified network model in which individual modules are represented as units with 1 second synaptic time constants. These long time constants reflect the strong within module feedback. The spike rate of module $i$ was given by the equation:

$$
\tau \frac{d x_{i}(t)}{d t}=-x_{i}(t)+\sum_{j} w_{i, j} r_{j}(t)+I_{i}(t)+P I_{i}(t)
$$

$$
r_{i}(t)=\tanh \left(x_{i}(t)\right)
$$

Where $\tau=1 \mathrm{~s}$, and the external input $/$ was equal to 1 during the sample period and 0 for all other time points. The network was trained using the FORCE algorithm ${ }^{10}$ to produce a persistent output along one dimension. On alternating training trials, broad transient photoinhibition given by $\mathrm{PI}(\mathrm{t})$ was applied immediately after the sample period. Training sought to produce a change in activity along the persistent mode during photoinhibition followed by a rapid recovery after the offset of photoinhibition. $\mathbf{b}$. We found that the trained network not only matched the target output, but also produced graded persistent activity in response to varying the amplitude of the external inputs. Magenta traces show the response of the network to broad photoinhibition illustrating the network's robustness to these particular perturbations. c. To illustrate the widespread nature of the photoinhibition, we plotted the change in activity resulting from photoinhibition for each module. $\mathbf{d}$. Finally, because of the long intrinsic modular time constants, we found that targeted photostimulation of small numbers of modules produced changes that persisted for several seconds. e. To assess whether targeted photostimulation cause sparse changes in network activity as in our data (Fig. 1g, Fig. 4a) we plotted the change in activity due to photostimulation for each module. The amplitude of excitation in the three targeted modules is significantly larger than in all other modules.

f. A hybrid discrete/continuous attractor model $^{8}$, can also produce rapid recovery to broad photoinhibition and persistent changes from targeted photostimulation. In this model, a fined-tuned 
374 approximately linear transfer function $f(x)=\frac{1.4}{1+e^{-(x-5) / .3}}(\mathbf{g})$ coupled with self-exciting and mutually 375 inhibiting $L$ (red) and $R$ (blue) neurons produces slow dynamics along the difference mode $[1,-1]$ and fast 376 dynamics along the $[1,1]$ direction. Large amplitude inhibition of both neurons produces a rapid recovery 377 (h) whereas weak excitation of one of the neurons produces a persistent change in activity (i). Because 378 this network is only composed of 2 units, it does not produce sparse and persistent responses, but a 379 network composed of many such modules would produce sparse responses. 
a

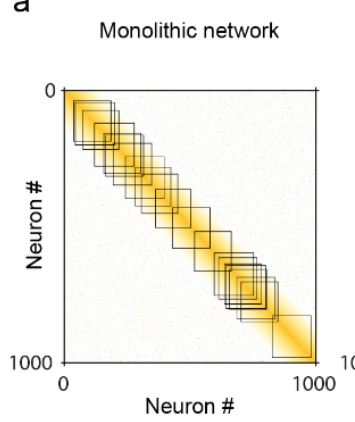

e

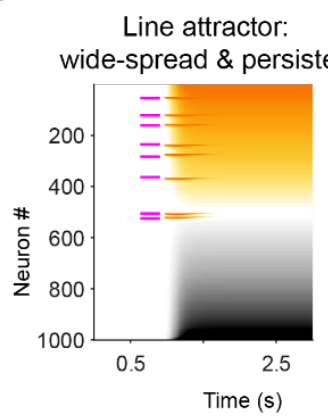
model networks

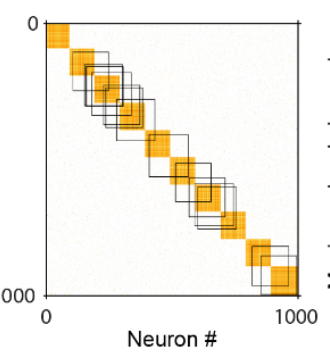

f

Discrete attractor:weakly wide-spread \& transient

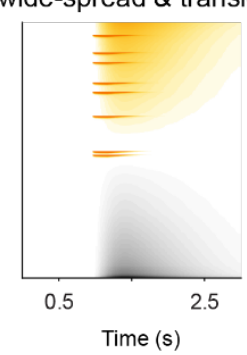

b

Local eigenvalues

c

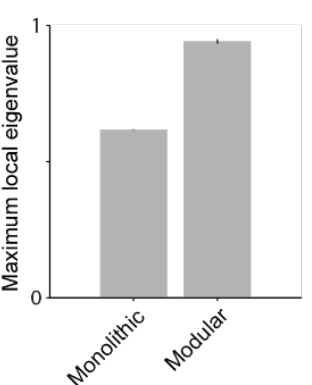

Local eigenvalues inferred connectivity

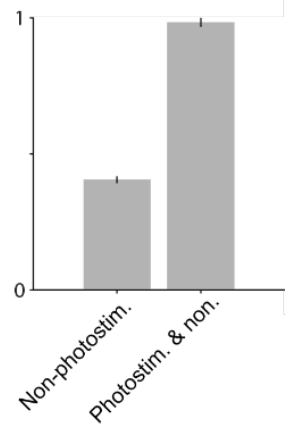

d

Connections from: $\mathrm{R}$
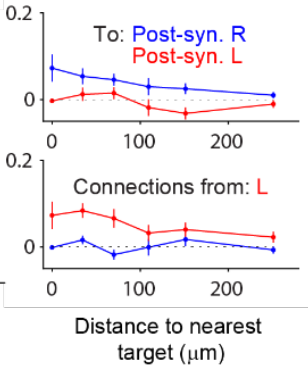

Extended Data Figure 10. Monolithic models. The key difference between the two model networks with locally biased connectivity in Figure $4 b-c$ is the presence of a single persistent mode of activity in the monolithic network and multiple distinct persistent modes in the modular network. More precisely, the monolithic network has a single mode with eigenvalue equal to 1 and the modular network has 10 such eigenvalues. We use this fact to develop an analysis aimed at determining which of these two models more closely resembles the connectivity inferred from the data in Figure 4d-e.

a. If we were to draw a box around one of the modules in Figure 4c, we would find that the maximum eigenvalue of this subnetwork is 1 , whereas an equivalent box in the monolithic model in Figure $4 \mathrm{~b}$ would have a maximum eigenvalue of 0.48 . To extend this "local eigenvalue" analysis to the inferred networks in Figure 4d-e we wouldn't know which neurons to draw this box around, so to make the analysis more general, we instead draw many boxes at random locations along the diagonal and calculate the maximum eigenvalue of subnetwork within the box. b. On average, the modular network has larger local eigenvalues 
394 (mean, 0.94, range, $0.84-1.0,75 \% \mathrm{Cl}$ ) than the monolithic network (mean, 0.62, range, $0.61-63,75 \%$

$395 \mathrm{Cl}$; error bars, s.e.m.).

396 c. For the inferred connectivity matrix (Fig. 4d-e), we identify all neurons within $70 \mu \mathrm{m}$ of a

397 photostimulation target whose activity was significantly perturbed by photostimulation $\left(p_{i}^{p g}<0.05\right.$,

398 methods), and calculate the maximum eigenvalue of this subnetwork. We find that the largest local

399 eigenvalue in networks trained to match photostimulation and non-photostimulation trials was similar to

400 the modular network in Fig. 4c (mean, 0.98 , range $0.75-1.27,75 \% \mathrm{Cl}$ ). Networks trained to match the

401 activity during non-photostimulation trials only (d) had a maximum eigenvalue that were similar to the

402 monolithic network (mean, 0.41 , range, $0.19-0.62,75 \% \mathrm{Cl}$ ).

403 e-f. Response of a line attractor (e) and discrete attractor (f) network (Methods) to targeted 404 photostimulation of 8 neurons. Due to widespread connectivity, perturbations of a small number of 405 neurons spread throughout both networks. 


\section{References}

408

409

410

411

412

413

414

415

416

417

418

419

420

421

422

423

424

425

426

427

1. Chen, T.-W. et al. Ultrasensitive fluorescent proteins for imaging neuronal activity. Nature 499, 295300 (2013).

2. Yang, W. et al. Simultaneous Multi-plane Imaging of Neural Circuits. Neuron 89, 269-284 (2016).

3. Packer, A. M. et al. Two-photon optogenetics of dendritic spines and neural circuits. Nat Meth $\mathbf{9}$, 1202-1205 (2012).

4. Rickgauer, J. P. \& Tank, D. W. Two-photon excitation of channelrhodopsin-2 at saturation. PNAS 106, 15025-15030 (2009).

5. GENIE project, Janelia Farm Campus, HHMI; Karel Svoboda (contact). 2015. Simultaneous imaging and loose-seal cell-attached electrical recordings from neurons expressing a variety of genetically encoded calcium indicators. CRCNS.org. $h t t p: / / d x$. doi.org/10.6080/K02R3PMN

6. Miri, A. et al. Spatial gradients and multidimensional dynamics in a neural integrator circuit. Nature Neuroscience 14, 1150-1159 (2011).

7. Li, N., Daie, K., Svoboda, K. \& Druckmann, S. Data and simulations related to: Robust neuronal dynamics in premotor cortex during motor planning. $h t t p: / / d x$. doi.org/10.6080/KO2R3PMN

8. Li, N., Daie, K., Svoboda, K. \& Druckmann, S. Robust neuronal dynamics in premotor cortex during motor planning. Nature 532, 459-464 (2016).

9. Inagaki, H. K., Fontolan, L., Romani, S. \& Svoboda, K. Discrete attractor dynamics underlies persistent activity in the frontal cortex. Nature 566, 212 (2019).

10. Sussillo, D. \& Abbott, L. F. Generating Coherent Patterns of Activity from Chaotic Neural Networks. Neuron 63, 544-557 (2009). 
Mice. Data are from eleven mice (age at the beginning of experiments, $70-150$ days). Eight mice were used for behavioral experiments. Two mice were used for characterization of the spatial resolution of photostimulation (Fig1. c, Extended Data Fig. 6) and one mouse for electrophysiology experiments

433 (Extended Data Fig. 5). All mice were from the CamK2a-tTA (JAX:007004) x Ai94(TITL-GCaMP6s) ${ }^{1}$ (JAX: 024104) transgenic line. Half of the mice were crossed with Emx1-Cre (JAX: 005628) and the other half were crossed with slc17a7 IRES Cre (JAX: 023527), both providing broad expression of GCaMP6s in excitatory cortical neurons.

Surgical procedures. All procedures were in accordance with protocols approved by the Janelia Institutional Animal Care and Use Committee. During surgical procedures mice were anesthetized using 1-2 \% Isoflurane. After surgery mice were given Buprenorphine $\mathrm{HCl}(0.1 \mathrm{ml}, 0.03 \mathrm{mg} / \mathrm{ml})$. Ketoprofen (0.1 $\mathrm{ml}, 0.1 \mathrm{mg} / \mathrm{ml}$ ) was provided on the day of surgery and for two days following surgery. Three millimeter 441 circular craniotomies were centered over ALM (2.5 mm anterior and $1.5 \mathrm{~mm}$ lateral from Bregma). Virus 442 (10 12 titer; AAV2/2 camKII-KV2.1-ChrimsonR-FusionRed; Addgene, plasmid \#102771) was injected $400 \mu \mathrm{m}$ 443 below the dura (4-10 sites, 20-30 $\mathrm{nl}$ each; or 2 sites, $100 \mathrm{nl}$ each), centered within the craniotomy and 444 spaced by $500 \mu \mathrm{m}$. The craniotomy was covered by a cranial window composed of 3 layers of circular glass 445 (total thickness, $450 \mu \mathrm{m}$ ). The diameter of the bottom two layers was $2.5 \mathrm{~mm}$ diameter. The top layer was $4463 \mathrm{~mm}$ or $3.5 \mathrm{~mm}$ and rested on the skull. The wind ow was cemented in place using cyanoacrylate glue and 447 dental acrylic (Lang Dental). A custom headbar was attached just anterior to the window using 448 cyanoacrylate glue and dental cement. After 3-7 days of recovery mice were placed on water restriction 449 (1 ml/day). Behavioral training started 3-5 days later. Coexpression of GCaMP6s and ChrimsonR was 450 confirmed in histological sections imaged using an inverted confocal microscope (Extended Data Fig. 1; Zeiss, LSM 880 Airyscan). 
Behavior. Behavioral training was performed as described previously ${ }^{2,3}$. Briefly, mice were presented with one of two auditory cues. Half of the mice were trained to discriminate between $3 \mathrm{kHz}$ and $12 \mathrm{kHz}$ pure tones and the other half were trained to discriminate between white noise or an equally weighted combination of pure tones with frequencies of $0.5,1,2,4,8$ and $16 \mathrm{kHz}$. No qualitative differences in ALM activity resulted from using different sets of auditory stimuli. During the sample epoch (1.25 seconds) white noise was played continuously whereas tones were played for five repetitions of 150 ms pulses with $100 \mathrm{~ms}$ inter-pulse intervals. During the sample and delay epochs, the lickports were out of reach of the mouse ${ }^{4}$. The lickports began moving towards the mouse 2.6 seconds after the start of the delay, arriving 3 seconds after the start of the delay. The arrival of the lickport served as a 'go cue' for the mice to begin licking. Mice were allowed to lick for reward 3 seconds after the start of the delay epoch.

Imaging and photostimulation experiments were started once the mice achieved performance of $>65 \%$, typically 4-6 weeks after the start of training. Statistical power analysis revealed that $65 \%$ performance maximizes our ability to detect changes in behavior caused by photostimulation. An exploratory round of experiments ( 3 mice, 33 sessions), in which 4 or 5 groups were photostimulated per experiment, showed small but robust changes in mouse behavior after photostimulation. Based on these preliminary experiments, in the second round of experiments we enhanced our ability to detect changes in behavior by increasing the number of trials per photostimulation group ( 5 mice, 51 sessions) at the expense of the number of photostimulation groups (two per session). Given the number of photostimulation trials per session (mean, 25 ; range, $13-38,75 \% \mathrm{Cl}$; per trial type), statistical power analysis indicated that

471 behavioral changes greater than $\pm 18 \%$ correspond to $p<0.05$ in single sessions.

472 Microscope. Two-photon imaging and two-photon photostimulation were performed using a custom 473 microscope ${ }^{4}$ with an additional of a photostimulation path, consisting of a $1040 \mathrm{~nm}$ pulsed laser (Fidelity 474 10, Coherent), a Pockels cell (Conoptics) for power modulation, and a pair of galvanometer mirrors 475 (Cambridge, 6215H) for beam positioning (Extended Data Fig. 2). Imaging was with $920 \mathrm{~nm}$ light 
476 (Chameleon Ultra II, Coherent) and a resonant scanner (Thorlabs). Imaging and photostimulation were

477 controlled by Scanimage 2016a (Vidrio).

478 The field of view was adjusted according to the spatial range of opsin expression (one of: $585 \mu \mathrm{m} \times 599 \mu \mathrm{m}$,

$479512 \times 512$ pixels, $30 \mathrm{~Hz} ; 814 \mu \mathrm{m} \times 732 \mu \mathrm{m}, 640 \times 640$ pixels, $24 \mathrm{~Hz} ; 968 \mu \mathrm{m} \times 822 \mu \mathrm{m}, 700 \times 700$ pixels, $22 \mathrm{~Hz})$.

480 Imaging was restricted to layer $2 / 3$ of anterior lateral motor cortex (ALM), 125-250 microns below the

481 surface of the brain.

482

483

484

485

486

487

488

489

490

491

492

493

494

495

Photostimulation and behavior experiments. Neurons were chosen for membership in a photostimulation group based on their selectivity in either the early delay, late delay or early response epochs. Selectivity of individual neurons was either determined based on activity in the first 30-70 trials in a session, or activity measured on the previous day. After defining 2-5 photostimulation groups (8 neurons each) for a session, the photostimulation targets were loaded into Scanlmage using custom Matlab software. Each neuron was photostimulated for $3 \mathrm{~ms}$ each, with 1 ms between photostimulation of different neurons (Extended Data Fig. 4). After all 8 neurons had been photostimulated $(8 x(3 \mathrm{~ms}+1 \mathrm{~ms})=32 \mathrm{~ms})$ the first neuron was photostimulated again until each neuron was photostimulated 10 times, for a total of $10 \times 32 \mathrm{~ms}=320 \mathrm{~ms}$. The power of the photostimulation beam at the sample was 100-150mW. Because this power was sufficient to excite GCaMP fluorescence, all imaging frames acquired during the photostimulation were discarded. Photostimuli were typically provided one second after start of the delay epoch (138/215 photostimulation groups), but in some experiments we photostimulated one second earlier (61/215 groups) or later (16/215 groups). Photostimulation and control trials were randomly interleaved, with photostimuli delivered on either $33 \%$ or $40 \%$ of trials.

Electrophysiology. Extracellular voltage was recorded in lightly anesthetized mice $(0.75 \%$ isoflurane) with cell-attached recordings. Signals were acquired using an Axopatch 700B amplifier (Molecular Devices) at $20 \mathrm{kHz}$ (http://wavesurfer.janelia.org). Electrodes with $10 \mathrm{M} \Omega$ impedance were filled with ACSF (in mM): 

in layer $2 / 3$ of primary motor cortex with 1 psi of positive pressure. Neurons were photostimulated with a train of $103-\mathrm{ms}$ spirals with a 28-ms inter-spiral interval at 50, 100 and $150 \mathrm{~mW}$.

Analysis of calcium-related fluorescence dynamics. Regions of interest (ROIs) corresponding to cell bodies were generated using a semi-automated algorithm ${ }^{5}$. Cell bodies were identified in images averaged across a session, and images in which all frames corresponding to correct lick-left trials were averaged and subtracted from an average of all frames from correct lick-right trials. These selectivity maps (Extended Data Fig. 3a) were used for choosing neurons for photostimulation and also for ensuring that all selective neurons were included in analysis. $f(t)=\Delta F(t) / F_{0}$ was calculated for each cell by defining baseline fluorescence $\left(F_{0}\right)$ as the average fluorescence during the pre-sample period averaged across all trials. Fluorescence traces were then separated by trial-type. The fluorescence trace at time $t$ of neuron $i$ on trial number $j$, trial type $k$ (left, $\mathrm{k}=\mathrm{L}$; right, $\mathrm{k}=\mathrm{R}$; both left and right, $\mathrm{k}=\mathrm{L} \& \mathrm{R}$ ) and photostimulation

511 group number $s$ (non-photostimulation, $s=$ non.; photostimulation of group $p g, s=p g$ ) is $f_{i, j}^{k, s}(t)$. In this

512 notation the fluorescence of neuron $i$ on the $j$ th lick right trial with photostimulation of group $p g$ is $f_{i, j}^{R, p g}$

Selectivity. Selectivity for trial type was used to choose neurons for photostimulation and to group neurons for analysis. Selectivity $S_{i}$ is the trial-averaged difference between fluorescence for left and right correct non photostimulation trials, around the go cue $\left(t_{c u e}\right)$ :

$$
S_{i}=\left\langle\left\langle f_{i, j}^{R, \text { non. }}(t)\right\rangle_{j=\text { correct trials }}-\left\langle f_{i, j}^{L, \text { non. }}(t)\right\rangle_{j=\text { correct trials }}\right\rangle_{t_{\text {cue }}-0.5 s>t>t_{\text {cue }}+0.5 s}
$$
photostimulation group pg on the ith neuron is (Fig. 1f,h): 


$$
\Delta_{i}^{L \& R, p g}(t)=\left\langle f_{i, j}^{L \& R, p g}\right\rangle_{j=\text { all trials }}-\left\langle f_{i, j}^{L \& R, \text { non. }}\right\rangle_{j=\text { alltrials }}
$$

521 To characterize the strength of photostimulation in each neuron and trial (Fig. 1g) we compared the time-

522 averaged fluorescence with and without photostimulation:

$$
\bar{f}_{i, j}^{L \& R, p g}=\left\langle f_{i, j}^{L \& R, p g}\right\rangle_{t_{p g}<t<t}+1 s \text { and } \bar{f}_{i, j}^{L \& R, n o n}=\left\langle f_{i, j}^{L \& R, n o n}\right\rangle_{t_{p g}<t<t}{ }_{p g}+1 s
$$

524 where $t_{p g}$ is the end of the photostimulus for group $p g . p_{i}^{p g}$ is the $\mathrm{p}$-value (two-tailed t-test) comparing

525 the distributions of $\bar{f}_{i, j}^{L \& R, p g}$ and $\bar{f}_{i, j}^{L \& R, n o n}$ for each neuron. We refer to all neurons that were within 20

$526 \mu \mathrm{m}$ of a photostimulation target with $p_{i}^{p g}<0.05$ as directly photostimulated. Neurons with $p_{i}^{p g}<0.05$

527 were plotted in Figure 1g ('perturbed').

528 The average selectivity of all directly photostimulated neurons is $\left\langle S_{i}^{\text {norm. }}\right\rangle_{i=\text { direct }}$ (Fig. 1f, gray shading; Fig.

529 1j; Fig. 2b, x-axis; Fig. 2c, bottom, y-axis). Here $S_{i}^{\text {norm. }}$ is the average selectivity, $S_{i}$, divided by the standard

530 deviation in fluorescence of neuron $i$ (z-scored), normalized to have $\mathrm{R}^{2}$ norm equal to one.

531 Persistence of photostimulation. To quantify the persistence of the change in activity produced by

532 photostimulation, we averaged the trial-averaged change in activity across all directly photostimulated

533 neurons, $\left\langle\Delta_{i}^{L \& R, p g}(t)\right\rangle_{i=\text { direct }}$ (Figure $1 \mathrm{~h}$ ). We define the decay time constant $\tau_{p g}$ as the time at which

$534\left\langle\Delta_{i}^{L \& R, p g}(t)\right\rangle_{i=\text { direct }}$ decays to $1 /$ e of its peak value. Because many photostimulation groups produce

535 changes that remain larger than 1/e of the peak after 3 seconds, we fit $\left\langle\Delta_{i}^{L \& R, p g}(t)\right\rangle_{i=\text { direct }}$ using

$$
\left\langle\Delta_{i}^{L \& R, p g}(t)\right\rangle_{i=\text { direct }}=a e^{-t / T_{\text {decay }}}\left(1-e^{-t / T_{\text {rise }}}\right) .
$$

$537 \tau_{p g}$ is the time when the fit decayed to 1/e (Figure 1h-j). Only photostimulation groups with 
included in analysis of persistence. In Figure $1 \mathrm{i}-\mathrm{j}$ data were binned in quintiles based on their value along the $\mathrm{x}$-axis.

542 from directly photostimulated neurons in group $p g$ with neuron $i$ was calculated as:

and $\mathrm{L}\left(\Delta L^{p g}\right)$ populations was calculated as the average of the coupling strength for all $\mathrm{R}$ and $\mathrm{L}$ (Fig. $2 \mathrm{~b}$,

$$
\Delta R^{p g}=\left\langle\text { Coupling } \operatorname{strength}_{i}^{p g} S_{i}^{n o r m}\right\rangle_{i=\left\{D_{i}^{p g}>30 \mu m, S_{i}^{n o r m} \cdot>0\right\}}
$$

$$
C_{i, l}^{R}=\left\langle\operatorname{Corr}\left(f_{i, j}^{R, \text { non. }}(t)-\left\langle f_{i, j}^{R, \text { non. }}(t)\right\rangle_{j=\text { all trials }}, f_{l, j}^{R, \text { non. }}(t)-\left\langle f_{l, j}^{R, \text { non. }}(t)\right\rangle_{j=\text { all trials }}\right)\right\rangle_{t}
$$


558 The average correlation of coupled neuron $i$ with photostimulation group $p g$ was $C_{i}^{R, p g}=\left\langle C_{i, l}^{R}\right\rangle_{l=\text { direct }}$. A

559 similar analysis was performed for left trials. Noise correlations for right and left trials were then averaged

560 (Fig. 2d, x-axis):

Avg. Noise Corr. $=C_{i}^{R, p g} / 2+C_{i}^{L, p g} / 2$

\section{Changes in behavioral performance.}

563 For each photostimulation group, we calculated the difference in correct response rate between 564 photostimulation trials and non-photostimulation trials separately for lick left and lick right trials. P values 565 were calculated by downsampling the non-photostimulation trials to match the number of 566 photostimulation trials. This process was repeated 10,000 times with replacement (Figure 3a, Extended

567 Data Fig. 8). To estimate the false-positive rate, we calculated 10,000 null-distributions of $p$-values from 568 the downsampled non-photostimulation trials (Figure 3b, gray line) and compared this to the distribution 569 of p-values from photostimulation trials (Figure 3b, black line) using the Kolmogorov-Smirnov test.

570 To relate changes in behavior to photostimulated changes in activity, we calculated 'activated population 571 selectivity' (Fig. 3c-f) as the overlap between the photostimulated change in activity $572\left\langle\Delta_{i}^{L \& R, p g}(t)\right\rangle_{t_{\mathrm{pg}}<t<t_{p g}+0.5 s}$ and trial-type selectivity, $S_{i}$. At the population level, the change in activity induced 573 by photostimulation was relatively sparse (Fig. 1d, Fig. 4a) and trial-to-trial variability was substantial. We 574 isolated the effects mediated by photostimulation from normal trial-to-trial variability. We first divided 575 the photostimulation trials into two halves, a testing and training set. We next determined a 576 photostimulation subspace $\mathbf{V}_{p g}$ via an SVD decomposition on the first half of the photostimulated data 577 for time points immediately following photostimulation:

$$
\left\langle\Delta_{i, \text { train }}^{L \& R g}(t)\right\rangle_{t_{\mathrm{pg}}<t}=\mathbf{U S} \mathbf{V}_{\text {train, }, p g}^{\prime}
$$


579 We then projected the change in activity from a separate subset of trials $\left(\left\langle\Delta_{i, t e s t}^{L \& R, p g}(t)\right\rangle_{t_{\mathrm{pg}}<t<t_{p g}+0.5 s}\right)$ onto 580 this photostimulation subspace to obtain the photostimulation vector (Fig. 3c-e).

$$
\text { Photostim. vector }{ }^{p g}=\sum_{m=1}^{M}\left(\sum_{i} V_{\text {train }, i, p g}^{m}\left\langle\Delta_{i, \text { test }}^{L \& R, p g}(t)\right\rangle_{t_{\mathrm{pg}}<t<t_{p g}+0.5 s}\right) V_{\text {train, }, \text {, pg }}^{m}
$$
$\left\langle\Delta_{i, t r a i n}^{L \& R, p g}(t)\right\rangle_{t_{\mathrm{pg}}<t<t_{p g}+0.5 s}$ was contained in the first mode of $\mathbf{V}_{p g}$ we limited analysis to this mode (i.e. observed for $M=2$. Activated population selectivity (Fig. $3 f ; x$-axis) was calculated as:

$$
\text { Activated pop. sel. }{ }^{p g}=\sum_{i} \text { Photostim. } \text { vector }_{i}^{p g} S_{i}
$$
population selectivity was computed for each photostimulation group. The same analysis was repeated by splitting non-photostimulation trials into two non-overlapping sets and treating one set as if it were from a photostimulation experiment. This sham photostimulation subset was selected to have the same number of trials as the actual photostimulation experiment (Fig. 3f, gray), and was further split in half into randomly sampled training and testing subsets for 10 repetitions as described above for the actual photostimulation experiments.

594 Network models. The spike rate of model neuron $i$ was determined by the equation:

$$
\tau \dot{r}_{i}(t)=-r_{i}(t)+\sum_{j} w_{i, j} G\left(r_{j}\right)(t)+I_{i}(t)
$$
$100 \mathrm{~ms}), G$ is the synaptic non-linearity and $I_{i}(t)$ is the external input. For the non-modular network with

598 local connectivity (Fig. 4b) the connectivity was $w_{i, j}=e^{-|i-j| / 70}$. For the monolithic attractor models 
599 (where all neurons contribute to the attractor) (Extended Data Fig. 10a, b) w was a rank-one matrix 600 constructed by taking the outer product of a Gaussian random $1000 \times 1$ norm-1 vector with itself. For the 601 line-attractor model (Extended Data Fig. 10a), the synaptic function was $G(x)=x$; the discrete attractor 602 model (Extended Data Fig. 10b) had $G(x)=0.1 \tanh (0.1 x)$. The modular attractor network (Fig. 4c) 603 consisted of 10 rank-one line-attractor networks, each composed of 100 neurons and $G(x)=x$. 604 Intermodular connections were sparse, with $96 \%$ of connections set to zero and the remaining 605 connections drawn randomly from a Gaussian distribution with mean zero. Short-term memory was 606 simulated by giving a brief input to each network along the network's primary eigenvector. 607 Photostimulation was simulated by injecting a brief current to 8 randomly chosen neurons.

608 Generating model connectivity by fitting to data. Connectivity matrices were trained to fit the 609 fluorescence activity of all individual neurons ${ }^{6}$. For each experimental session with $N$ recorded neurons we fit the $N \times N$ matrix $w_{i, j}$ to reproduce the fluorescence of each neuron. To account for the time-course 611 of calcium-sensitive fluorescence we approximated the network equation to be:

$$
\tau \frac{d}{d t}\left\langle f_{i, j}^{k, s}(t)\right\rangle_{j}=-\left\langle f_{i, j}^{k, s}(t)\right\rangle_{j}+\Sigma_{l} w_{i, l} \tanh \left(\left\langle f_{l, j}^{k, s}(t)\right\rangle_{j}\right)+\tilde{\kappa}_{G C a M P 6 s} \otimes\left(I_{i}^{k}(t)+I_{i}^{s}(t)\right)
$$

613 We used the approximate kernel $\tilde{\kappa}_{\text {GCaMP6s }}=e^{-t \backslash 0.7 s}$, which reflects the slow decay component of the 614 GCaMP6s response to a burst of spikes (Extended Data Fig. 7). External sensory $\left(I_{i}^{k}\right)$ and photostimulation

$615\left(I_{i}^{s}\right)$ currents were step functions that were only active during the sample and photostimulation epochs, 616 respectively. Fitting was done using a recursive least-square algorithm ${ }^{6,7}$ in which all weights onto a given 617 neuron are tuned at each time step to minimize the difference between its modeled and experimentally 618 observed fluorescence activity. Activity from different trial types ( $k=$ left \& $k=$ right) and photostimulation 619 conditions ( $\mathrm{s}=$ non., s = pg $1 \ldots \mathrm{pg} \mathrm{N}$ ) were fit sequentially. Each fit was iterated 30 times, which produced 620 high-quality fits (Median Pearson correlation $=0.69$, range $0.25-0.92,75 \% \mathrm{Cl}$ ). For each session, two 
bioRxiv preprint doi: https://doi.org/10.1101/623785; this version posted April 30, 2019. The copyright holder for this preprint (which was not certified by peer review) is the author/funder. All rights reserved. No reuse allowed without permission.

621 separate fits were obtained, one to capture only non-photostimulation trials and the other to capture

622 both photostimulation and non-photostimulation trials.

623 
1. Madisen, L. et al. Transgenic mice for intersectional targeting of neural sensors and effectors with high specificity and performance. Neuron 85, 942-958 (2015).

627 2. Guo, Z. V. et al. Procedures for Behavioral Experiments in Head-Fixed Mice. PLoS ONE 9, e88678

628 (2014).

629

3. Inagaki, H. K., Inagaki, M., Romani, S. \& Svoboda, K. Low-Dimensional and Monotonic Preparatory Activity in Mouse Anterior Lateral Motor Cortex. J. Neurosci. 38, 4163-4185 (2018).

631

4. Peron, S. P., Freeman, J., Iyer, V., Guo, C. \& Svoboda, K. A Cellular Resolution Map of Barrel Cortex Activity during Tactile Behavior. Neuron 86, 783-799 (2015).

5. Chen, T.-W. et al. Ultrasensitive fluorescent proteins for imaging neuronal activity. Nature 499, 295300 (2013). Memory. Neuron 90, 128-142 (2016).

637 7. Sussillo, D. \& Abbott, L. F. Generating Coherent Patterns of Activity from Chaotic Neural Networks. Neuron 63, 544-557 (2009). 